\title{
Interacting vorticity waves as an instability mechanism for MHD shear instabilities
}

\author{
E. Heifetz ${ }^{1,2}$, J. Mak ${ }^{1} \dagger$, J. Nycander ${ }^{2}$ and O. M. Umurhan ${ }^{3,4}$ \\ ${ }^{1}$ Department of Geophysics \& Planetary Sciences, Tel Aviv University, Tel Aviv, 69978, Israel \\ ${ }^{2}$ Department of Meteorology, Stockholm University, Stockholm, Sweden \\ ${ }^{3}$ NASA Ames Research, Space Sciences Division, Mail Stop N-245-3, Moffett Field, CA 94043 \\ ${ }^{4}$ SETI Institute, 189 Bernardo Ave., Suite 100, Mountain View, CA 94043
}

(Received DATE UPDATED)

The interacting vorticity wave formalism for shear flow instabilities is extended here to the magnetohydrodynamic (MHD) setting, to provide a mechanistic description for the stabilising and destabilising of shear instabilities by the presence of a background magnetic field. The interpretation relies on local vorticity anomalies inducing a non-local velocity field, resulting in action-at-a-distance. It is shown here that the waves supported by the system are able to propagate vorticity via the Lorentz force, and waves may interact; existence of instability then rests upon whether the choice of basic state allows for phase-locking and constructive interference of the vorticity waves via mutual interaction. To substantiate this claim, we solve the instability problem of two representative basic states, one where a background magnetic field stabilises an unstable flow and the other where the field destabilises a stable flow, and perform relevant analyses to show how this mechanism operates in MHD.

Key Words: ${ }^{* * *}$ do this on submission website ${ }^{* * *}$

\section{Introduction}

Shear flows are ubiquitous in fluid systems, and shear flow instability, nonlinear development and its transition into turbulence remains an active area of research to the present day. We focus here on is magnetohydrodynamic (MHD) shear instabilities, relevant to astrophysical systems such as, for example, the solar tachocline, the magnetopause, and atmospheres of hot exoplanets. In particular, we are interested in the physical mechanisms leading to ideal parallel shear instabilities in MHD.

The argument generally is that, in the presence of a background magnetic field that has a component parallel to the background flow, fluid instabilities have to do work to bend field lines, thus the presence of a background magnetic field should be stabilising. This is generally found to be true in planar geometry when the background magnetic field is uniform (e.g., Chandrasekhar 1981; Biskamp 2003). However, this argument does not account for the observed destabilisation of hydrodynamically stable flows in the presence of spatially varying background magnetic fields (e.g., Stern 1963; Kent 1966, 1968; Chen \& Morrison 1991; Tatsuno \& Dorland 2006; Lecoanet et al. 2010), or the fact that a uniform field can destabilise some wavenumbers that are hydrodynamically stable (e.g., Kent 1966, 1968; Ray \& Ershkovich 1983). Further, in two-dimensional spherical

$\dagger$ Email address for correspondence: julian.c.l.mak@googlemail.com. 


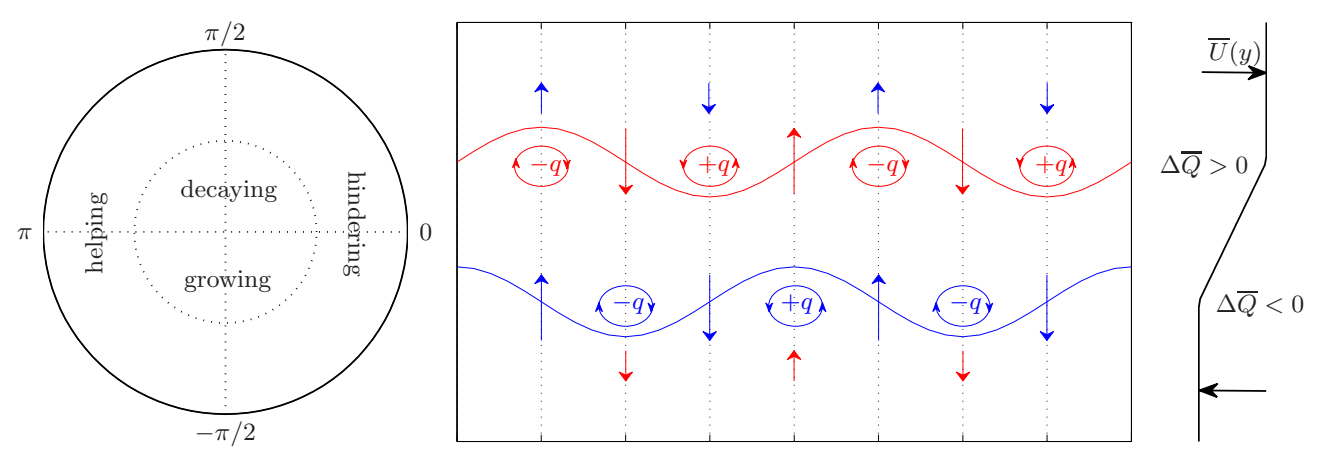

FIgURE 1. A pictorial representation of the wave interaction mechanism for the case of two Rossby waves. Here, $q \sim-\Delta \bar{Q} \eta$, where $q$ is vorticity, $\eta$ is displacement, and $\Delta \bar{Q}$ is the magnitude of the vorticity jump of the background flow profile. Taking into account the sign of $\Delta \bar{Q}$ at each jump, the $q$ anomalies resulting from the $\eta$ distribution are labelled accordingly. The top wave has a self-induced propagation to the left relative to the mean flow, and vice versa for the bottom wave, and both waves propagating counter to the mean flow. The waves interact with the other via the non-local velocity field induced by local vorticity anomalies, and the induced velocities decay with distance from vorticity anomalies, represented here by the length of the arrows. The waves may be held steady owing to counter-propagation relative to the mean flow, together with the mutual interaction between the waves, resulting in a phase-locked configuration. The waves can then constructively interfere and lead to instability. A phase difference regime diagram is given on the left, where the phase-difference is defined as $\Delta \epsilon=\epsilon_{2}-\epsilon_{1}$, the lower wave displacement relative to the upper wave; the configuration here has $\Delta \epsilon=-\pi / 2$.

geometry (no radial motion), a flow close to the observed solar differential rotation is hydrodynamically stable, but is destabilised by the presence of an azimuthal background magnetic field varying in latitude (e.g., Gilman \& Fox 1997; Gilman \& Cally 2007). To reconcile these contrasting effects, the negative-energy wave interpretation (e.g., Cairns 1979), based on wave resonance and energetic arguments, is sometimes invoked (e.g., Ruderman \& Belov 2010). The aim here is to provide an intuitive mechanistic interpretation that reconciles the contrasting influences of the magnetic field on MHD shear instabilities.

We present here an interpretation of shear instabilities in terms of interacting vorticity waves. This interpretation includes as a special case the Counter-propagating Rossby Waves (CRW) mechanism (e.g., Bretherton 1966; Hoskins et al. 1985), a mechanism wellknown in geophysical fluid dynamics, and has been argued by Baines \& Mitsudera (1994) to be an equivalent approach to the negative-energy wave explanation for shear instability. A basic schematic of the mechanism is recalled here for the Rossby wave case in figure 1 with a description of the mechanism given in the caption.

The main ingredients required for instability in this interpretation are phase-locking and constructive interference, achieved via counter-propagation of waves relative to the mean flow, action-at-a-distance by the non-local velocity field induced by local vorticity anomalies, and an appropriate phase-shift between the two waves. Normal mode instability may also occur whenever the waves are phase-locked with relative phase-difference (defined here in terms of wave displacement) satisfying $\Delta \epsilon \in(-\pi, 0)$. If the self-propagation speed of each wave is large compare to its local mean flow, the action-at-a-distance interaction should hinder the waves' propagation speed to maintain phase-locking; this occurs when $\Delta \epsilon \in(-\pi / 2,0)$, and generally characterise long wavelength dynamics. By contrast, the self-propagation speed of short wavelength waves counter to the mean flow is generally weak, hence instability is obtained when the waves help each other's propagation to 
overcome the background flow while growing, and this occurs when $\Delta \epsilon \in(-\pi,-\pi / 2)$. Essentially, the mechanism may be summarised as "the induced velocity field of each Rossby wave keeps the other in step, and makes the other grow" (Hoskins et al. 1985). For more details, we refer the reader to the recent review of Carpenter et al. (2013) and references within.

In the case of figure 1, we have illustrated the fundamental mechanism using Rossby waves, but this is not the only possibility. As long as we have wave modes that counterpropagate against the mean flow and they propagate vorticity, it is perfectly possible for a configuration displayed in figure 1 to occur. Harnik et al. (2008), Rabinovich et al. (2011) and Guha \& Lawrence (2013) have shown that, in the context of shear instabilities in stratified fluids, displacement of an interface leads to an induced buoyancy field, that in turn induces an appropriate vorticity field via baroclinic torque, thus Rossby-gravity waves may also propagate vorticity. When two vorticity and/or density interfaces are present, the interaction of the relevant wave modes leads to instability of the KelvinHelmholtz type (two vorticity interfaces; e.g, Drazin \& Reid 1981, Baines \& Mitsudera 1994), Holmboe type (essentially one vorticity and one density interface; Holmboe 1962, Baines \& Mitsudera 1994), and the Taylor-Caulfield type (two density interfaces; Tavlor 1931, Caulfield 1994). For recent reviews and studies of shear instabilities in stratified fluids and their interpretation in terms of wave interactions, see Balmforth et al. (2012), Carpenter et al. (2010, 2013) and Guha \& Lawrence (2013).

We show here that a similar interpretation holds in MHD. Wave displacement changes the magnetic field configuration, and since the Lorentz force is generally rotational, this in turn generates vorticity anomalies, so Alfvén waves may propagate vorticity. We can thus use the vorticity wave interaction framework as an interpretation for MHD shear instabilities. Furthermore, this dynamical framework explains why we have stabilisation or destabilisation by the magnetic field: the choice of basic state and parameter values affects the properties of the wave modes, and the presence of instability depends on whether supported wave modes can phase-lock and achieve mutual amplification.

Previous works on this topic have, for elucidation purposes, mainly considered piecewiselinear basic states with interfacial wave solutions of the form $q(x, y, t)=\hat{q} \delta(y-L) \mathrm{e}^{\mathrm{i} k(x-c t)}$, where $y=L$ is the location of 'jump' in the profile. If one can show that the vorticity generation is non-zero only at these jumps, then interfacial wave solutions are exact solutions. A small number of jumps in the basic state results in a dispersion relation that is a low order algebraic equation with closed form solutions. Further analysis of the solutions may be carried out in a relatively straight-forward manner (e.g., Rabinovich et al. 2011). One notable exception to this exactness in the hydrodynamic setting is the Charney problem (e.g., Heifetz et al. 2004b), where the presence of differential rotation $\beta$ results in non-localised vorticity generation. A similar phenomenon occurs for the MHD case. Although this does not pose a problem for the numerical computation of the eigenvalues and eigenfunctions, extra care is required when interpreting the results and the physical mechanism. This subtlety is explained in detail here, and the regimes where the interfacial wave assumption is a reasonable approximation to the full solution are explored accordingly for the instability problems we consider.

The layout of this article is follows. We provide the mathematical set up in $\S 2$, and explain how even simple Alfvén waves propagate vorticity via the Lorentz force. Additionally, we demonstrate the non-local nature of vorticity generation. For conceptual understanding of how waves in the system propagate vorticity, we consider the dynamics of interfacial waves in $\S 3$. To demonstrate the instability mechanism, we solve the instability problem for two piecewise-linear basic states, for comparison with previous work employing the interacting vorticity wave formalism, and to test the performance of the 
interfacial wave assumption. In $\S 4$, we consider the case where a background magnetic field stabilises the flow, taking the background flow to be the Rayleigh profile demonstrated in figure 1 together a uniform background magnetic field. We first give details for the numerical method we employ, then analyse in some detail the full solution, providing plots of eigenfunctions and showing how the schematic in figure 1 is modified by MHD effects. Analytic solutions resulting from the interface assumption are derived, compared with the full solutions, and analysed accordingly. We give a similar account in $\S 5$ for the case where a linear shear flow is destabilised by a spatially varying background magnetic field. We conclude and discuss our results in $\S 6$.

\section{Mathematical formulation}

The crux of the mechanism displayed in figure 1 is that waves supported by the system and choice of basic state propagates vorticity, and their interaction leads to instability. In this section, we provide the general mathematical formulation, and explain how even simple Alfvén waves, supported when the background flow and field are uniform, may propagate vorticity via the Lorentz force. We consider the dynamics of waves when the flow and/or field is sheared in the next section.

\subsection{Two dimensional MHD and action of Lorentz force}

We are interested here in ideal MHD instabilities. The homogeneous, incompressible MHD equations are

$$
\begin{gathered}
\frac{\partial \boldsymbol{u}}{\partial t}+\boldsymbol{u} \cdot \nabla \boldsymbol{u}=-\frac{1}{\rho_{0}} \nabla p+\frac{1}{\mu_{0} \rho_{0}} j^{*} \times \boldsymbol{B}^{*}, \\
\frac{\partial \boldsymbol{B}^{*}}{\partial t}+\boldsymbol{u} \cdot \nabla \boldsymbol{B}^{*}=\boldsymbol{B}^{*} \cdot \nabla \boldsymbol{u}, \\
\nabla \cdot \boldsymbol{u}=0, \quad \nabla \cdot \boldsymbol{B}^{*}=0 .
\end{gathered}
$$

Here, $\boldsymbol{j}^{*}=\nabla \times \boldsymbol{B}^{*}$ is the current. In Cartesian co-ordinates, an analogue of Squire's theorem holds (see e.g., Hughes \& Tobias 2001, and discussion within), thus we may formulate the problem in two-dimensions. The domain is taken to be the $(x, y)$-plane, with periodicity in $x$, and as yet unspecified in $y$. The incompressibility condition allows us to write the velocity $\boldsymbol{u}$ and magnetic field $\boldsymbol{B}=\boldsymbol{B}^{*} / \sqrt{\mu_{0} \rho_{0}}$ (where $\mu_{0}$ is the permeability of free-space and $\rho_{0}$ is the constant density) in terms of a streamfunction $\psi$ and magnetic potential $A$, defined here as $\boldsymbol{u}=\boldsymbol{e}_{z} \times \nabla \psi$ and $\boldsymbol{B}=\boldsymbol{e}_{z} \times \nabla A$. From this, the vorticity $q=\boldsymbol{e}_{z} \cdot \nabla \times \boldsymbol{u}$ and current $j=\boldsymbol{e}_{z} \cdot \nabla \times \boldsymbol{B}$ satisfy the relations $q=\nabla^{2} \psi$ and $j=\nabla^{2} A$, and equations (2.1) take the equivalent form

$$
\frac{D q}{D t}=\nabla \cdot(j \boldsymbol{B}), \quad \frac{D A}{D t}=0 .
$$

To see how the Lorentz force $\nabla \cdot(j \boldsymbol{B})$ results in rotational motion, we suppose we have, for argument sake, $B_{0} \boldsymbol{e}_{x}$ with $B_{0}=$ const $>0$, and

$$
j\left(x_{0}, y_{0}\right) \boldsymbol{e}_{z}=j_{0} \boldsymbol{e}_{z}, \quad j\left(x_{1}, y_{1}\right) \boldsymbol{e}_{z}=j_{1} \boldsymbol{e}_{z},
$$

where $j_{1}>j_{0}, x_{1}>x_{0}, y_{1}=y_{0}$; this is a scenario where $\partial\left(j b_{x}\right) / \partial x>0$. Now, in general, the Lorentz term is $\boldsymbol{F}=\boldsymbol{j} \times \boldsymbol{B}$, and, with the right-hand-screw convention, the current distribution above produces

$$
\boldsymbol{F}\left(x_{0}, y_{0}\right)=F_{0} \boldsymbol{e}_{y}, \quad \boldsymbol{F}\left(x_{1}, y_{1}\right)=F_{1} \boldsymbol{e}_{y},
$$


(a) $B_{x}=B_{0}>0$

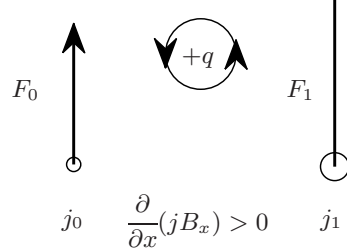

(b) $B_{y}=B_{0}>0$

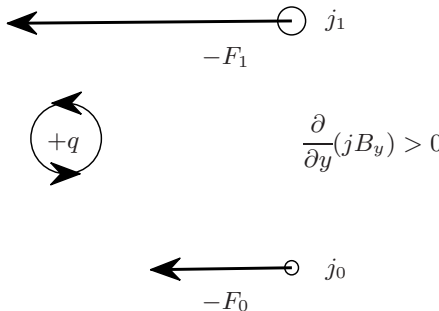

FiguRE 2. A pictorial representation of how the vorticity is generated by the Lorentz term $\boldsymbol{j} \times \boldsymbol{B}=\nabla \cdot(\boldsymbol{B} \boldsymbol{B})$ in two-dimensional incompressible MHD. For $j_{1}>j_{0}$, this generates the appropriately signed vorticity anomalies.

with $F_{1}>F_{0}>0$. A material line connecting $\left(x_{0}, y_{0}\right)$ and $\left(x_{1}, y_{1}\right)$ is rotated anticlockwise, i.e., this gives us a positive vorticity anomaly; this is consistent with positive forcing in equation (2.2). This is shown pictorially in figure 2( $(a)$.

Analogously, we could instead take $B_{0} \boldsymbol{e}_{y}$, and the above current distribution but taking $x_{1}=x_{0}$ and $y_{1}>y_{0}$ (together with the analogous assumptions); this is the scenario where $\partial\left(j b_{y}\right) / \partial y>0$. This results in

$$
\boldsymbol{F}\left(x_{0}, y_{0}\right)=-F_{0} \boldsymbol{e}_{y}, \quad \boldsymbol{F}\left(x_{1}, y_{1}\right)=-F_{1} \boldsymbol{e}_{y},
$$

where $F_{1}>F_{0}$, and this may be seen to produce also anti-clockwise motion of a material line. This is again consistent with equation (2.2), and is shown in figure 2(b). Then, in the linear case, the resulting action is a superposition of the above scenarios and others like it.

\subsection{Linearisation and Alfvén wave dynamics}

We now linearise (2.2) about a general basic state $\bar{U}(y) \boldsymbol{e}_{x}$ and $\bar{B}(y) \boldsymbol{e}_{x}$ (and thus $\bar{Q}=$ $-\partial \bar{U} / \partial y$ and $\bar{J}=-\partial \bar{B} / \partial y$ ), which results in the system of equations

$$
\begin{aligned}
\left(\frac{\partial}{\partial t}+\bar{U} \frac{\partial}{\partial x}\right) q & =-\frac{\partial \bar{Q}}{\partial y} \frac{\partial \psi}{\partial x}+\bar{B} \frac{\partial j}{\partial x}+\frac{\partial \bar{J}}{\partial y} \frac{\partial A}{\partial x}, \\
\left(\frac{\partial}{\partial t}+\bar{U} \frac{\partial}{\partial x}\right) A & =\bar{B} \frac{\partial \psi}{\partial x} .
\end{aligned}
$$

The cross-stream displacement $\eta$ is given by

$$
v=\frac{\partial \psi}{\partial x}=\left(\frac{\partial}{\partial t}+\bar{U} \frac{\partial}{\partial x}\right) \eta
$$

and, substituting this into (2.6b) and integrating results in the relation

$$
A=\bar{B} \eta+A_{0}(y),
$$

where we will take the non-advective contribution $A_{0}$ associated with non-conservative effects to be zero for the rest of this work.

For simple Alfvén waves (e.g., Biskamp 2003), we take the case $\bar{U}=0$, and $\bar{B}=$ constant $>0$ without loss of generality. With this, the governing equations become $\partial q / \partial t=\bar{B}(\partial j / \partial x)$ and $\partial \eta / \partial t=\partial \psi / \partial x$. We observe that $\eta \sim A$ from relation (2.8), i.e., a positive displacement is correlated with a positive $A$ anomaly. Furthermore, since $\nabla^{2} A=j$ and the Laplacian is a negative definite operator, this implies the relation $\eta \sim-j$. 

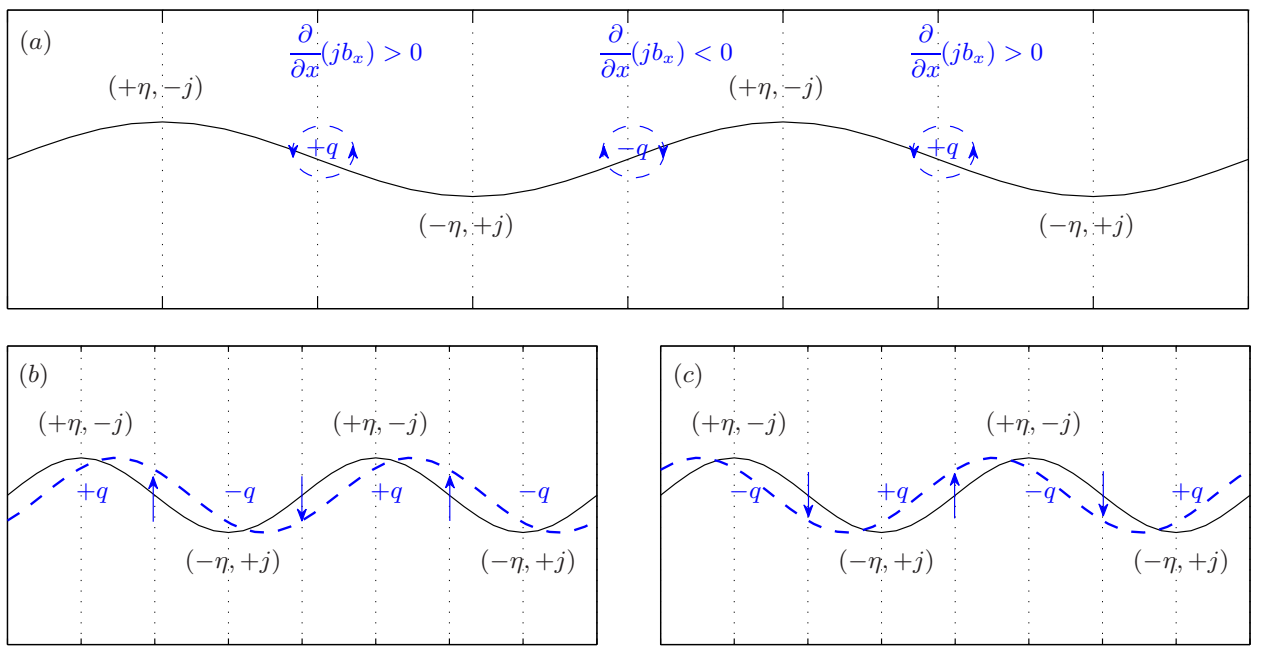

Figure 3. Pictorial representation for wave dynamics associated with $\bar{B}(\partial j / \partial x)$, which is the linearised form of $\partial\left(j b_{x}\right) / \partial x$, and includes simple Alfvén waves as a particular case. See text for description.

Suppose now we have some wave structure, as in figure 3(a). Associated with such a wave structure is a $j$ distribution, anti-correlated with $\eta$ in this set up. From this, since $\bar{B}>0$, the distribution of $j$ leads to positive and negative vorticity anomalies in between the wave crests and troughs depending on the sign of $\partial j / \partial x$, resulting in the configuration in figure $3(a)$. To maintain a wave structure, appropriate vorticity anomalies must exist in phase or anti-phase with $\eta$ to counteract the action of the vorticity distribution at the notes. Alternatively, the configuration in figure 3 $(a)$ may be seen as a superposition of equal amplitude right and left going waves (e.g., appendix B of Harnik et al. 2008). If $q$ is in phase with $\eta$ as in panel (b), then this gives a wave propagating to the right. On the other hand, if $q$ is in anti-phase with $\eta$ as in panel $(c)$, this gives the wave propagating to the left. It may be checked from the resulting linearised equations that, if we consider modal solutions of the form $\mathrm{e}^{\mathrm{i} k(x-c t)}$ (we take the $y$-wavenumber to be zero for simplicity), since the resulting dispersion relation for simple Alfvén waves is $c^{2}= \pm \bar{B}$ in this set up, $c>0$ implies we have $\eta \sim-j \sim q$, whilst for $c<0, \eta \sim-j \sim-q$. These relations are consistent with the schematic presented in figure 3 .

\subsection{Green's function formulation}

Wave interaction via action-at-a-distance may be represented mathematically by a Green's function formalism, employed by previous authors (e.g., Harnik et al. 2008). Noting that $j=\nabla^{2} A$ and $\psi=\nabla^{2} q$, and taking the domain to be $x$-periodic, a Fourier transform leads to

$$
j=\left(-k^{2}+\frac{\partial^{2}}{\partial y^{2}}\right) A, \quad q=\left(-k^{2}+\frac{\partial^{2}}{\partial y^{2}}\right) \psi
$$

where $k$ denotes the $x$-wavenumber. (2.9) may be formally inverted to give

$$
\left(\begin{array}{l}
A(y) \\
\psi(y)
\end{array}\right)=\int G\left(y, y^{\prime}\right)\left(\begin{array}{l}
j\left(y^{\prime}\right) \\
q\left(y^{\prime}\right)
\end{array}\right) \mathrm{d} y^{\prime},
$$

where $G\left(y, y^{\prime}\right)$ is a Green's function chosen to satisfy the boundary conditions. Then, with the governing equations (2.6a), (2.7), and substituting for the appropriate terms 
using relations (2.8) and (2.10), the problem is completely specified in terms of $q$ and $\eta$, where all intermediate effects from changes in $A$ and $j$ are implicit.

For piecewise-linear profiles we consider in the subsequent sections, we note this approach of using Green's functions guarantees the continuity of the total pressure and displacement everywhere. The kinematic condition for the continuity of the interface is already invoked in (2.7). From the $y$-component of the linearised momentum equation, we observe that the inverted $v$ and $b_{y}$ from $q$ and $j$ are guaranteed to be continuous by properties of the Green's function, so the total pressure $p$ is also continuous since $\bar{U}$ and $\bar{B}$ are continuous.

\subsection{Generation of vorticity away from interfaces}

If the background profiles are piecewise-linear, and, without loss of generality, a 'jump' is located at $y=L$, then $q \sim \hat{q} \delta(y-L)$ is exact if it may be shown that vorticity generation is only non-zero at that location. One notable exception however is the Charney problem, (e.g., Heifetz et al. 2004b) where vorticity generation is non-local, and we demonstrate here this occurs in the MHD case also. To show this, we take the Laplacian of (2.6b), and the resulting linearised equation for $j$ is given by

$$
\left(\frac{\partial}{\partial t}+\bar{U} \frac{\partial}{\partial x}\right) j=\left(\frac{\partial \bar{Q}}{\partial y}+2 \bar{Q} \frac{\partial}{\partial y}\right) \frac{\partial A}{\partial x}+\bar{B} \frac{\partial q}{\partial x}-\left(\frac{\partial \bar{J}}{\partial y}+2 \bar{J} \frac{\partial}{\partial y}\right) \frac{\partial \psi}{\partial x} .
$$

Then, even if $(\partial \bar{Q} / \partial y, \partial \bar{J} / \partial y)=(\Delta \bar{Q}, \Delta \bar{J}) \delta(y-L), j$ and $q$ are not necessarily $\delta$-functions in $y$ since $\bar{Q}(\partial A / \partial y)$ or $\bar{J}(\partial \psi / \partial y)$ may not be zero for $y \neq L$. Thus interfacial wave solutions fails to capture all the dynamics since we are setting to zero contributions away from the interfaces, and we do not know a priori whether the neglected contributions are significant to the dynamics. Further, non-local generation of vorticity implies that critical layers will play a role in the dynamics, much like the Charney problem (Heifetz et al. 2004b) and the second example considered in Rabinovich et al. (2011) for the stratified problem.

Although we will choose $q$ and $\eta$ as the fundamental variables for the bulk of the discussion, this is not the only possibility. It turns out there are some numerical advantages in utilising $q$ and $j$ as the variables when computing for the full numerical solutions. The two choices are of course equivalent as far as the full solution is concerned. A brief discussion of the $(q, j)$ equations is given in appendix $\mathrm{B}$ to discuss how they differ when interfacial wave dynamics are the focus.

\section{Interfacial wave dynamics}

We compute the wave modes supported in this MHD setting assuming a single interface for conceptual progress, to see how we may expect waves to propagate vorticity schematically. We consider an unbounded $y$-domain; the Green's function for this setting is given by

$$
G\left(y, y^{\prime}\right)=-\frac{1}{2 k} \mathrm{e}^{-k\left|y-y^{\prime}\right|} .
$$

We take piecewise-linear $\bar{U}$ and $\bar{B}$ (thus piecewise-constant $\bar{Q}$ and $\bar{J}$ ), with

$$
\frac{\partial \bar{Q}}{\partial y}=\Delta \bar{Q} \delta(y-L), \quad \frac{\partial \bar{J}}{\partial y}=\Delta \bar{J} \delta(y-L) .
$$


Equations (2.6a) and (2.7) then become

$$
\begin{aligned}
& \left(\frac{\partial}{\partial t}+\mathrm{i} k \bar{U}\right) q=\mathrm{i} k[-\Delta \bar{Q} \psi \delta(y-L)+\bar{B} j+\Delta \bar{J} A \delta(y-L)], \\
& \left(\frac{\partial}{\partial t}+\mathrm{i} k \bar{U}\right) \eta=\mathrm{i} k \psi .
\end{aligned}
$$

This set of resulting equations bears some formal resemblance to the analogous stratified problem (e.g., Harnik et al. 2008); this formal analogy is detailed in appendix $\mathrm{A}$

We consider solutions of the form

$$
q=\hat{q} \mathrm{e}^{-\mathrm{i} k c t} \delta(y-L), \quad j=\hat{j} \mathrm{e}^{-\mathrm{i} k c t} \delta(y-L) .
$$

With the inversion relation (2.10), the Green's function (3.1) for this domain, and taking modal solutions for $\psi$ and $A$, we obtain the relations

$$
\psi(L)=-\frac{\hat{q}}{2 k}, \quad A(L)=-\frac{\hat{j}}{2 k} .
$$

Using $A=\bar{B} \eta$ from (2.8), this results in $\hat{j}=-2 k \bar{B}(L) \eta(L)$. Together, (3.3) becomes

$$
(\bar{U}-c) \hat{q}=\frac{\Delta \bar{Q}}{2 k} \hat{q}-2 k \bar{B}\left(\bar{B}-\frac{\Delta \bar{J}}{2 k}\right) \eta, \quad(\bar{U}-c) \eta=-\frac{1}{2 k} \hat{q},
$$

where all the functions associated with the basic state are taken to be evaluated at $y=L$.

Now, combining the two equations in (3.6) and solving the resulting quadratic equation in $(\hat{q} / \eta)$, the eigenstructure and the dispersion relation reads

$$
\hat{q}^{ \pm}=2 k\left(c^{ \pm}-\bar{U}\right) \eta^{ \pm}, \quad\left(c^{ \pm}-\bar{U}\right)=-\frac{\Delta \bar{Q}}{4 k} \pm \sqrt{\left(\frac{\Delta \bar{Q}}{4 k}\right)^{2}+\bar{B}\left(\bar{B}-\frac{\Delta \bar{J}}{2 k}\right)} .
$$

We will call these modes generalised Rossby-Alfvén waves. The 'plus' branch is the one where the plus sign is taken, and analogously for the 'minus' branch. We observe that, when the waves are neutral, the plus branch is associated with a wave propagating to the right, relative to the mean flow, and vice-versa for the minus branch.

\subsection{A physical description of interfacial wave dynamics}

The eigenstructure (3.7) tells us how $q$ is related to $\eta$ directly from the equations. To understand the relation in terms of changes in the magnetic field configuration and how it results in vorticity anomalies and wave propagation, it is informative to consider how

the individual components act. In (3.7), the presence of the $\Delta \bar{Q}, \bar{B}^{2}$ and $\bar{B} \Delta \bar{J}$ terms is associated with the first, second and third term on the right hand side of equation (2.6a) respectively. The first term of the three is the standard Rossby wave mechanism, for which the restoring force comes from the background vorticity gradient. The wave propagation is that already described in figure 1. For the second term, this is essentially the Alfvén wave case described in the previous subsection; the eigenstructure relation (3.7) may be seen to be consistent with the schematic presented in figure 3.

The third term on the right hand side of $(2.6 a)$ is $(2.6 a)$ is

$$
\frac{\partial \bar{J}}{\partial y} \frac{\partial A}{\partial x}=\frac{\partial \bar{J}}{\partial y} b_{y}=\bar{B} \frac{\partial \bar{J}}{\partial y} \frac{\partial \eta}{\partial x}
$$

upon using the relation (2.8). We note $b_{y}(\partial \bar{J} / \partial y)$ is the linearised form of $\partial\left(j b_{y}\right) / \partial y$. We show in figure 4 a schematic depiction of how the $b_{y}(\partial \bar{J} / \partial y)$ term acts, with the details 


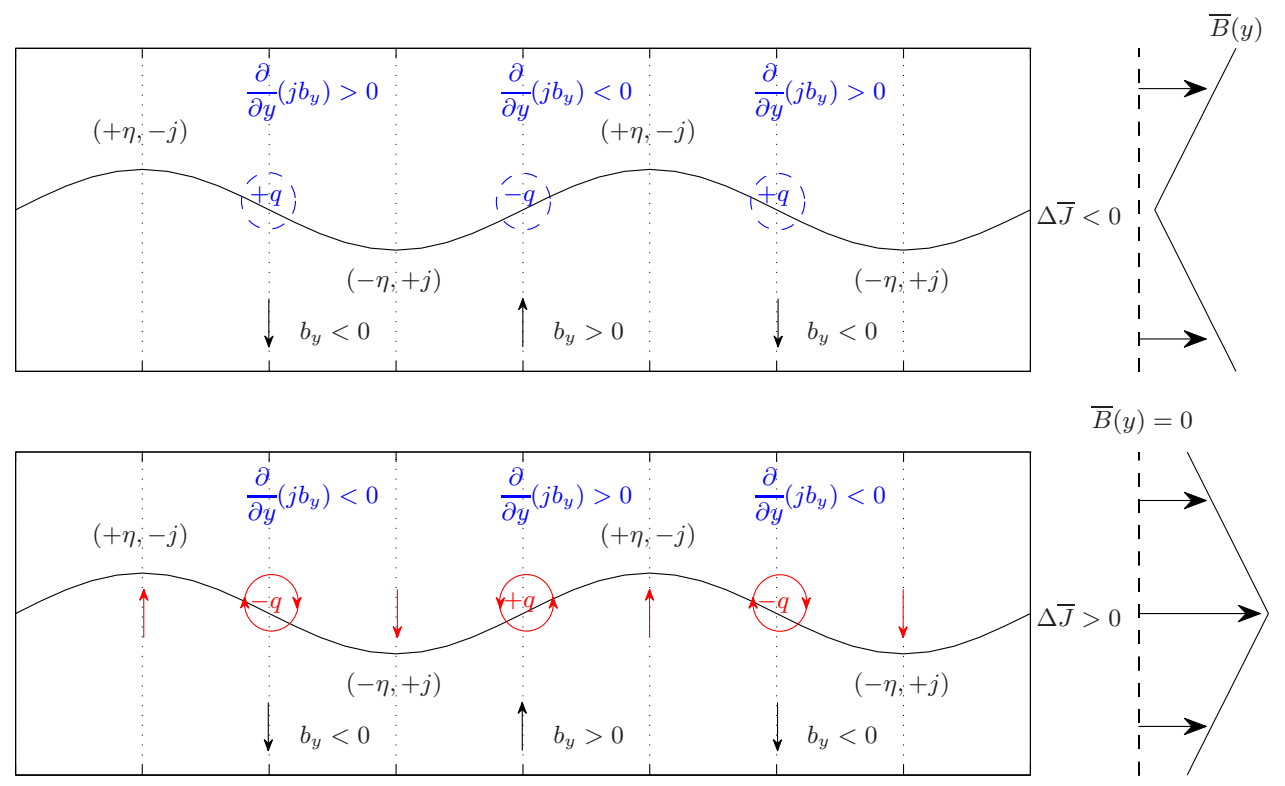

Figure 4. Pictorial representation for wave dynamics associated with $b_{y}(\partial \bar{J} / \partial y)$, the third term on the right hand side of (2.6a), which is the linearised form of $\partial\left(j b_{y}\right) / \partial y$. Taking $\bar{B}>0$, we have again $\eta \sim-j$ as before (see paragraph just after equation 2.8). Associated with the current anomalies is a distribution of $b_{y}$, given in black in both panels. Now, in $(a), \Delta \bar{J}<0$, and so this gives the appropriate $\partial\left(j b_{y}\right) / \partial y$ in blue, leading to vorticity anomalies depicted in blue. In this case, this is exactly analogous to the scenario depicted in figure 3 and leads to wave propagation. In $(b), \Delta \bar{J}>0$, and gives the appropriate vorticity distribution in red. In this case, there is no configuration of $q$ in phase or anti-pahse with $\eta$ that preserves the wave structure, and this leads to a prediction of an apparent local instability.

given in the caption. From the figure, we see that the sign of the vorticity anomalies generated depends on the sign of $\bar{B} \Delta \bar{J}$. In the case where $\bar{B} \Delta \bar{J}<0$, we see that both the second and third term in (2.6a) generate a vorticity distribution that contributes to wave propagation; see figure $3(a)$.

When $\bar{B} \Delta \bar{J}>0$, however, there is competition between the $\bar{B}(\partial j / \partial x)$ and $b_{y}(\partial \bar{J} / \partial y)$ terms since they generate vorticity of the opposite sign. The contribution from the latter term always dominates in the long-wave limit, leading to the situation depicted in figure $4(b)$. This scenario suggests an instability of the background magnetic field profile even in the absence of a background flow. Although magneto-static profiles can suffer ideal MHD instabilities (e.g., Ch. 19, Goldston \& Rutherford 1995), we note here that, since we are in planar geometry and we take $\bar{B}(y)$, this basic state is stable in ideal MHD by a theorem due to Lundquist (1951) because there the magnetic field lines possesses no curvature. This prediction of instability at the local level when the state is globally stable presumably stems from the fact that we are considering isolated $\delta$-function solutions for the purposes of elucidating the physics linking vorticity anomalies with wave displacement. The neglected contributions and the resulting mutual interactions will presumably suppress this apparent instability. For completeness, we note that, for the hydrodynamic stratified setting, the schematic presented in figure $4(b)$ is formally similar to an interpretation of Rayleigh-Taylor instability (e.g., Harnik et al. 2008).

In the presence of a background shear flow, however, instability is possible. As highlighted in Stern (1963), taking a profile with $\bar{B} \Delta \bar{J}<0$ everywhere in the domain results 
in instability when the background flow is stable in the hydrodynamic setting. Similar investigations in planar geometry of the destabilising nature of the magnetic field have mainly considered profiles where $\bar{B} \Delta \bar{J}<0$ in the domain, so here, for one of the examples, we also take a basic state that satisfies this condition.

\section{Unstable profile stabilised by uniform magnetic field}

Having demonstrated how we expect waves to propagate vorticity anomalies, we now consider two instability problems to demonstrate the instability mechanism, one where the field stabilises an unstable flow, and the other where the field destabilises a stable flow. Both basic states are chosen so that there is only one non-dimensional parameter, given by $M=\tilde{B} / \tilde{U}$, where the tildes denotes the relevant Alfvén speed and velocity scales. We note that, from linear analysis, there is a stability theorem which states that $|\bar{B}| \geqslant|\bar{U}|$ pointwise everywhere guarantees the absence of exponentially growing instabilities (e.g., Hughes \& Tobias 2001); the basic states are tailored so that the case $M \geqslant 1$ is equivalent to the aforementioned condition, with $M=1$ the case where we have equality.

We first provide details to the numerical method we employ to obtain our full solutions, then analyse these solutions and explain how the instability mechanism detailed in figure 1 is modified by MHD effects. To test the validity of taking only a small number of interfaces, we consider the approximated problem where only two interfaces are taken; closed form solutions may be obtained from the resulting low order algebraic system, and these are compared with the full solutions accordingly.

\subsection{Numerical method}

The governing equations depend on the choice of variables we employ for the numerical scheme. If we describe the dynamics in terms of $q$ and $\eta$, the governing equations are (2.6a) and (2.7). Upon using $j=\nabla^{2} A=\nabla^{2}(\bar{B} \eta)$, where we have used the identity (2.8), and with $\psi=\int q\left(y^{\prime}\right) G\left(y, y^{\prime}\right) \mathrm{d} y^{\prime}$, the resulting system of equations (2.6a) and (2.7) may be written in discretised form as

$$
\frac{\partial}{\partial t}\left(\begin{array}{l}
q \\
\eta
\end{array}\right)=-\mathrm{i} k\left(\begin{array}{cc}
\overline{\mathrm{U}}+\overline{\mathrm{Q}}^{\prime} \mathrm{G} & -\overline{\mathrm{B}}^{2}\left(-k^{2} \mathbf{I}+\mathrm{D}_{2}\right)+2 \overline{\mathrm{BJ}} \mathrm{D}_{1} \\
-\mathrm{G} & \overline{\mathrm{U}}
\end{array}\right)\left(\begin{array}{l}
q \\
\eta
\end{array}\right),
$$

where, for example $\bar{U}=\bar{U}\left(y_{i}\right) \times \mathrm{I}$, a prime denotes a $y$-derivative, and $\mathrm{D}_{1,2}$ are the appropriate discretised differential operators for $y$-derivatives. Taking uniform grid spacing $\Delta y$, we take for example $\partial \bar{Q} / \partial y=\Delta \bar{Q} / \Delta y$ at the $y_{j}$ entry when $\partial \bar{Q} / \partial y=\Delta \bar{Q} \delta\left(y-y_{j}\right)$. If a quantity $\bar{J}$ is discontinuous at $y_{j}$, we take $\bar{J}\left(y_{j}\right)=\left[\bar{J}\left(y_{j-1}\right)+\bar{J}\left(y_{j+1}\right)\right] / 2$. The discretised Green's function is given by (Harnik et al. 2008)

$$
\mathrm{G}_{m, n}=-\frac{\Delta y}{2 k} \mathrm{e}^{-k\left|y_{m}-y_{n}\right|}
$$

and this is a dense matrix. The system may be advanced in time accordingly if one is considering an initial value problem in the context of non-modal instabilities (e.g., Constantinou \& Ioannou 2011). For $(q, \eta)=(\tilde{q}, \tilde{\eta}) \mathrm{e}^{-\mathrm{i} k(x-c t)}$, the eigenvalues and eigenvectors of the system (4.1) are the normal mode solutions.

It turns out it is numerically more stable to solve the problem in $(q, j)$ variables, thus we couple equation (2.6a) with (2.11) instead of (2.7). A similar manipulation using $A=\int j\left(y^{\prime}\right) G\left(y, y^{\prime}\right) \mathrm{d} y^{\prime}$ results in

$$
\frac{\partial}{\partial t}\left(\begin{array}{l}
q \\
j
\end{array}\right)=-\mathrm{i} k\left(\begin{array}{cc}
\overline{\mathrm{U}}+\overline{\mathrm{Q}}^{\prime} \mathrm{G} & -\overline{\mathrm{B}}-\overline{\mathrm{J}}^{\prime} \mathrm{G} \\
-\overline{\mathrm{B}}+\overline{\mathrm{J}}^{\prime} \mathrm{G}+2 \overline{\mathrm{J}} \mathrm{G}^{\prime} & \overline{\mathrm{U}}-\overline{\mathrm{Q}}^{\prime} \mathrm{G}-2 \overline{\mathrm{Q}} \mathrm{G}^{\prime}
\end{array}\right)\left(\begin{array}{l}
q \\
j
\end{array}\right),
$$


where, in this case, $G^{\prime}$ is the derivative of the Green's function, with discretised form defined to be

$$
\mathrm{G}_{m, n}^{\prime}= \begin{cases}+(\Delta y / 2) \mathrm{e}^{-k\left(y_{m}-y_{n}\right)}, & y_{m}>y_{n}, \\ -(\Delta y / 2) \mathrm{e}^{+k\left(y_{m}-y_{n}\right)}, & y_{m}<y_{n}, \\ 0, & y_{m}=y_{n} .\end{cases}
$$

The value of 0 is taken at $y_{m}=y_{n}$ because the wave supported on $y_{m}$ does not induce any $u$ or $b_{x}$ at $y_{m}$. The lack of derivative operators in this latter matrix (4.3) results in a lower condition number when compared to the $(q, \eta)$ formulation in the test examples we have considered. The two formulations are of course equivalent, and the relevant eigenfunctions may be obtained from both formulations; here, the numerical results presented were obtained from solving (4.3).

Results presented here have been subject to domain size and resolution tests. A domain size of $y \in[-5,5]$ was employed. A resolution of $\Delta y=10^{-2}$ (in non-dimensional units) was found to be sufficient for modes away from marginality, while for modes close to marginality, a resolution of $\Delta y=5 \times 10^{-3}$ was employed instead to avoid the appearance of spurious instabilities. The linear algebra problem was solved using the eig(A) command in MATLAB, and we pick out the mode with the largest imaginary part. Sample tests shows the most unstable eigenvalues obtained are well separated from the rest of the spectrum and, further, occurs in conjugate pairs (a more general result for ideal instabilities which may be shown via consideration of the adjoint form of the governing equation; see, e.g., Drazin \& Reid 1981).

\subsection{Basic state and full numerical solution}

We consider first the Rayleigh profile as the background flow, with a uniform background magnetic field. In dimensional form, we take

$$
\bar{U}(y)=\left\{\begin{array}{ll}
\Lambda L, & y>L, \\
\Lambda y, & |y|<L, \\
-\Lambda L, & y<-L,
\end{array} \quad \bar{B}(y)=B_{0} .\right.
$$

For this problem, $\bar{J}=0$ and $\partial \bar{J} / \partial y=0$. Scaling by

$$
\tilde{B}=B_{0}, \quad \tilde{T}=\frac{1}{\Lambda}, \quad \tilde{L}=L, \quad \tilde{U}=\Lambda L,
$$

the resulting non-dimensional parameter is $M=\tilde{B} / \tilde{U}$, a ratio of the typical Alfvén velocity and the shear velocity, effectively a measure of the field strength, and $\bar{B} \rightarrow M$ and $j \rightarrow M j$ in (4.3) (as well as 4.1) upon rescaling. $M \geqslant 1$ corresponds to the regime where there are no linear, normal mode instabilities. The linear stability properties of this basic state has been studied by Ray \& Ershkovich (1983), who focused on computation of the growth rates of the instabilities via a shooting method, however not on the structure of the eigenfunctions.

We show in figure 5 the growth rates of the full solution $\left(k c_{i}\right)_{\text {full }}$ over parameter space; we note that $c_{r}=0$ for all unstable modes here, a result arising from the symmetries possessed by the basic state. The calculated growth rates are in agreement with the results documented in Rav \& Ershkovich (1983) after taking into account the different scalings used. The explanation for the shape of the instability region is that, at $M=0$, there is no phase-locking for short waves since they are too slow to overcome the background advection. At moderate $M$, these short waves are now sufficiently fast (from the nondimensional version of 3.7) and overcome the background advection, can become phase- 


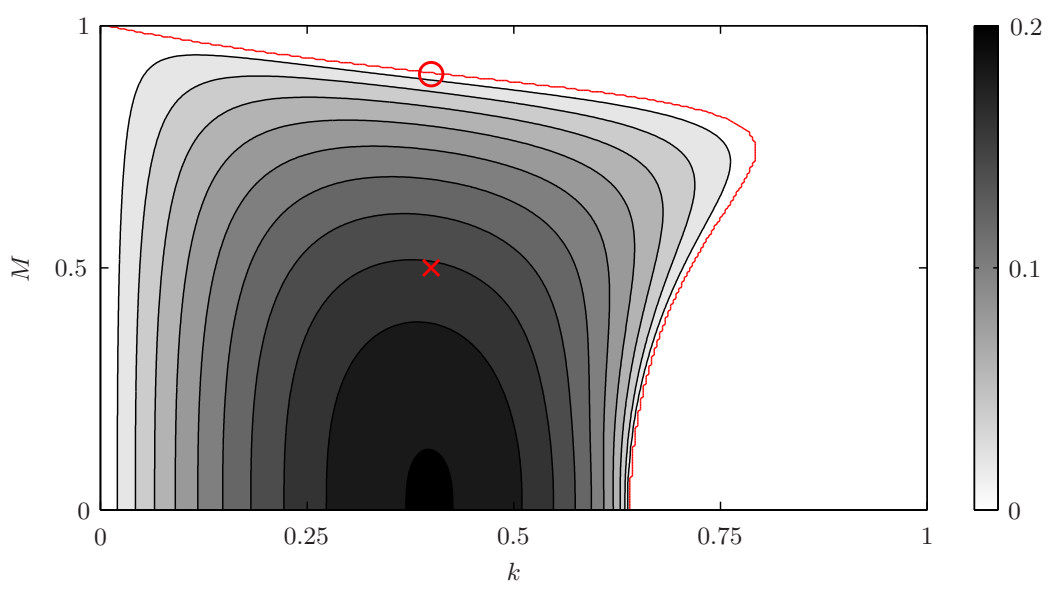

FIGURE 5. Growth rates $\left(k c_{i}\right)_{\text {full }}$ of the full numerical solution associated with the basic state (4.5). Here, $c_{r}=0$ for all unstable modes. The red cross and circle correspond to the parameter location associated with the eigenfunction displayed in figure $6(a, b)$ respectively.

locked, and constructively interfere. At large enough $M$, all waves become too fast, and phase-locking is not possible.

We show in figure 6 two eigenfunctions, one configuration that is generic for parameter values away from the stability boundary, and one at the same wavenumber, but at increased $M$ and is a sample configuration for a case close to marginality; the parameter choices are given by the red cross and circle in figure 5 respectively.

In figure $6(a)$, the eigenfunction configuration is generic in that the dominant contributions to vorticity comes from the two interfaces at $y= \pm 1$, and the contributions in the $|y| \neq 1$ region are comparatively small (note the colour axis scales of the corresponding sub-panels). The outer two vorticity contributions have a phase-shift that is close to $\pi / 2$ (0.25 in the normalised units used in the diagram), and the middle tilted structure has vorticity contributions that have opposite signs in between two like-signed vorticity contributions from the interface. The vorticity eigenfunction is, going from top to bottom, in anti-phase, in quadrature and in phase with displacement, i.e., $q \sim(-\eta,-\mathrm{i} \eta,+\eta)$ respectively. The outer two waves are really counter-propagating vorticity waves, and the inner wave resembles a standing wave. We have further carried out a decomposition of the vorticity eigenfunction into its constituents using equation (2.6a); in this case, the non-zero terms are $-(\partial \psi / \partial x)(\partial \bar{Q} / \partial y)$ and $\bar{B}(\partial j / \partial x)$, divided by $\bar{U}-c$. At the interface locations, the vorticity contribution comes from both terms, with the $-(\partial \psi / \partial x)(\partial \bar{Q} / \partial y)$ term being the dominant contribution, and the effect of the $\bar{B}(\partial j / \partial x)$ contribution is to alter the magnitude and the phase-difference between the two waves.

Figure 6(a) may roughly be represented schematically as figure 7 . With the configuration depicted, we see that the presence of the middle standing wave counteracts the effects of the vorticity anomalies associated with the outer counter-propagating waves. If we were to make the interface assumption, we neglect the contributions away from the interfaces and remove this centre contribution, and we thus expect to over-estimate (i), the growth rates, and (ii), the propagation speed, the region where phase-locking is possible and thus the size of the instability region. We would expect the over-estimation to be most significant when the field strength is large, and for short waves. We expect this for short waves because the interaction decreases exponentially with wavenumber, 
(a)

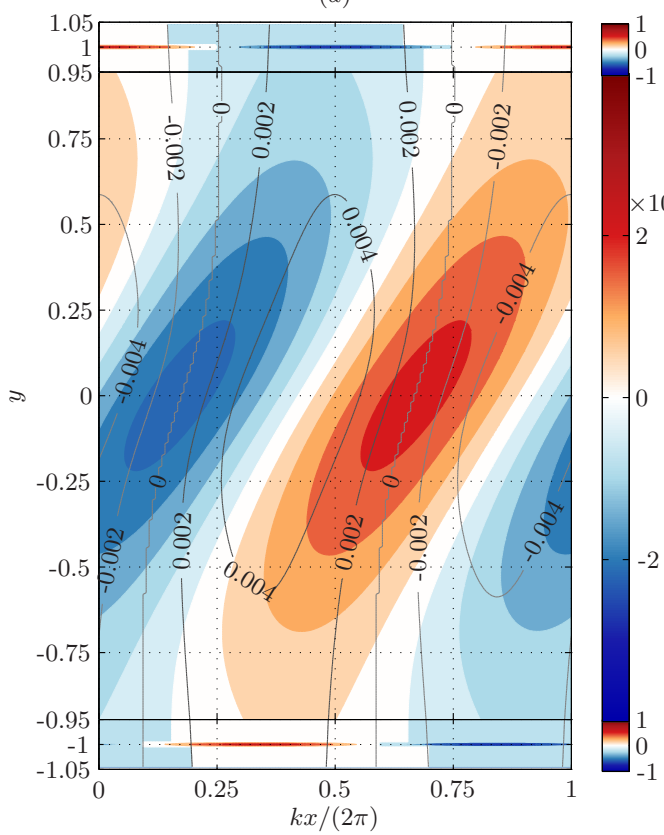

(b)

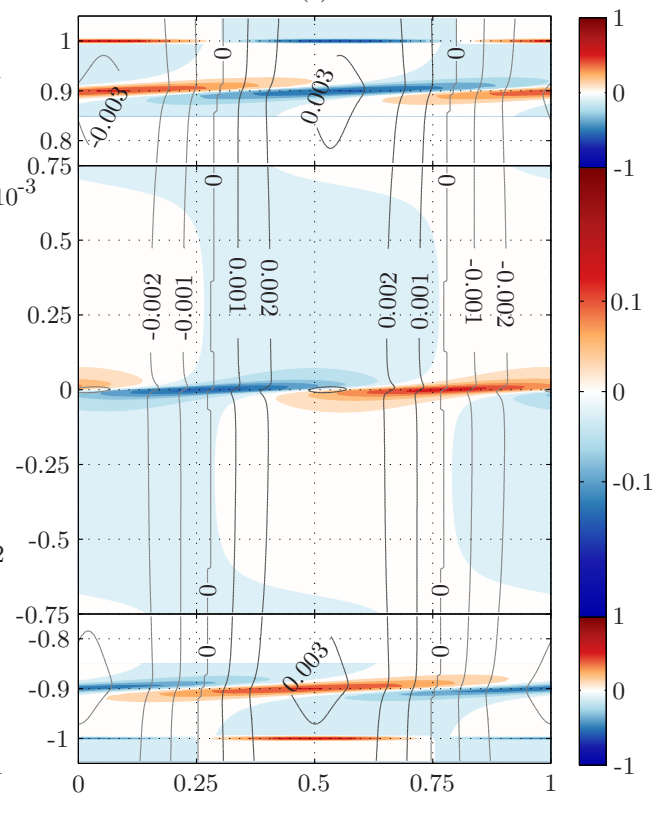

FiguRE 6. Representative eigenfunctions (normalised by the maximum absolute value of $q$ ) of the profile given in (4.5), both for $k=0.4$, but with (a) $M=0.5$, and (b) $M=0.9$. The red and blue shading are for positive and negative $q$ respectively, and $\eta$ is plotted as labelled contours; note the difference in the colour scales used between the sub-panels. The $y$-scale is continuous between the panels (note the displacement contours are continuous in magnitude) but is not linear for display purposes.

so the overall constructive interference between the counter-propagating waves is weaker for short waves than long waves, and it is short waves that are too slow to overcome the background advection.

The phase relation for the middle contribution $q \sim-\mathrm{i} \eta$ may be obtained from considering the vorticity equation (2.6a) . Away from $y= \pm 1$, we have, for this problem,

$$
q=\frac{M^{2} j}{\left(\bar{U}-c_{r}\right)-\mathrm{i} c_{i}} .
$$

We may suppose that $q$ is likely to be maximised at the location where $\bar{U}-c_{r}=0$, which is $y=0$ here since $c_{r}=0$, resulting in the relation $q \sim \mathrm{i} M^{2} j / c_{i}$. From relation (2.8), we have $j \sim \nabla^{2} \eta$, and so $q \sim-\mathrm{i} M^{2} \eta / c_{i}$, as required. This is analogous to what was found in the stratified setting for Rabinovich et al. (2011). The effect of the critical level is expected to be more pronounced when we are near marginality.

As we approach marginality via increasing $k$ and/or increasing $M$, the tilting of the middle structure increases, becomes thinner in the cross-stream extent, and eventually splits into three tilted structures. A sample case where we approach marginality by increasing $M$ is shown in figure $6(b)$. The first thing to note is that vorticity generation is not just significant at the location where $\bar{U}(y)-c_{r}=0$, but also at around $y= \pm M$. The mechanistic interpretation for instability and stabilisation however is not significantly altered. There is perhaps some cancellation of the contributions due to the tilted structures, but, schematically, we still have two counter-propagating waves with a stand- 


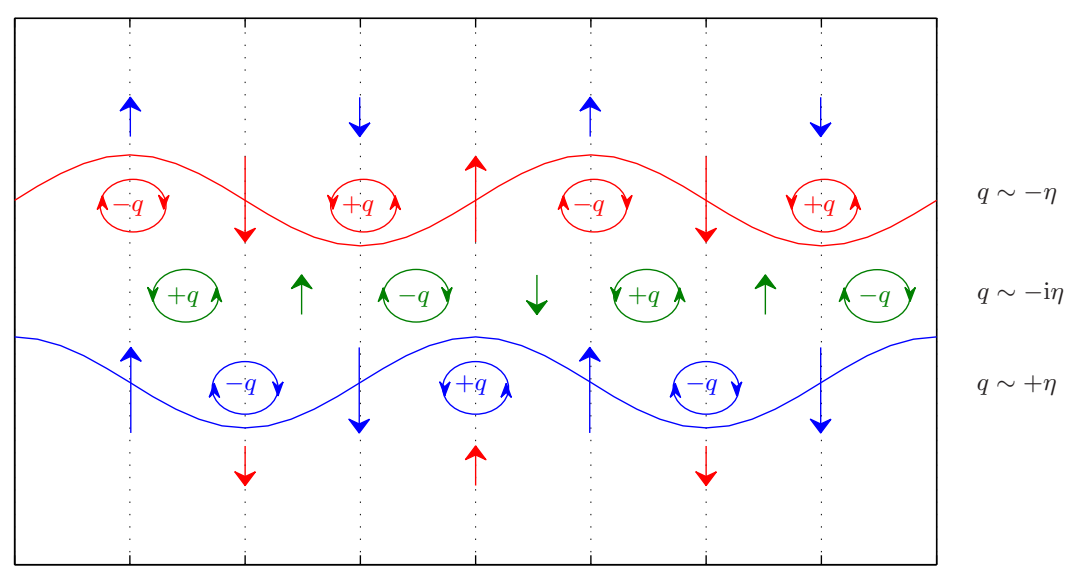

FiguRE 7. Schematic of how the critical layer contribution plays a role in the dynamics. Without the centre contribution denoted by the green parts, the dynamics of the two waves $(q \sim-\eta$ and $q \sim+\eta)$ are as in figure1. The centre wave acts as a standing wave with $q \sim-\mathrm{i} \eta$, with a resulting vorticity configuration as depicted in the figure. Then it is seen that the extra contributions acts against the vorticity anomalies associated with the counter-propagating waves, and is thus a stabilising effect.

ing wave in between that counteracts the vorticity contributions associated with the counter-propagating waves, again as in figure 7.

The structures at $y= \pm M$ are perhaps not too surprising once we note that Alfvén waves are non-dispersive and have non-dimensional phase speed $c_{\mathrm{a}}= \pm M$ in our setting, regardless of the wave number. Thus, from a physical point of view, we have forced, short wavelength standing Alfvén waves since $\bar{U}(y)-c_{\mathrm{a}}=0$ at these locations. From a mathematical point of view, we recall that, equivalently, the modal problem with general basic state in two-dimensional incompressible MHD in non-dimensional form is governed by the second-order differential equation

$$
\frac{\mathrm{d}}{\mathrm{d} y}\left(S^{2}(y) \frac{\mathrm{d} \eta}{\mathrm{d} y}\right)-k^{2} S^{2}(y) \eta=0, \quad S^{2}(y)=(\bar{U}(y)-c)^{2}-M^{2} \bar{B}^{2}(y) .
$$

If we take the view that critical levels are where the governing eigenvalue differential equations break down, the critical levels can occur when If we take the view that critical levels are where the governing eigenvalue differential equations break down, the critical levels can occur when $\bar{U}(y)-c=0$ and also where $\bar{U}(y)-c= \pm M \bar{B}(y)$, with the latter associated with Alfvén waves. The appearance of multiple critical levels is not an isolated feature in MHD, and appears for example in shear flow problems on the $f$-plane (e.g., appendix B of Lott 2003).

The second point to note for figure $6(b)$ is the change in the phase-shift of the counterpropagating components compared to figure 6 $(a)$, even though the same wavenumber was chosen. The stabilisation does not solely come from increasing the strength of the centre contribution, unlike in one of the examples considered by Rabinovich et al. (2011). By changing the field strength, we change both the strength of the critical layer contribution and the wave properties at the interfaces. Although the critical layer contribution is now stronger, the phase-shift between the vorticity contributions of the outer waves are also approaching an anti-phase configuration, and both effects contribute to the reduction in growth rate. It is also interesting to recall that, when the two outer vorticity contributions 
are in anti-phase, this implies that the corresponding displacement is in phase (e.g., Heifetz et al. 2004a), as seen in the $\eta$ eigenfunction. In other words, the whole region is undulating as one; this is physically consistent in that, as we increase the field strength, the magnetic field imparts more 'stiffness' to the fluid, and the fluid is forced to undulate as a whole.

As a final point, figure $6(b)$ is generic for eigenfunctions near marginal stability in the sense that, as $c_{i}$ goes to zero, the tilted structures become thinner and the configuration goes to one where there is no longer constructive interference due to the vorticity associated with the counter-propagating modes being in phase or in anti-phase. We have shown here the case where vorticity becomes increasingly out of phase, but of course they may also become increasingly in phase depending on where we are on the stability boundary. There is an intermediate region where the stabilisation is solely due to the strengthening of the centre contribution, but this is a somewhat special case and, generically, both the changes to phase-shift and strengthening of the critical layer contributes to the neutralisation of the instability.

\subsection{Interfacial wave dynamics}

From figure 6(a), we observe that the contribution in the centre can be small, so we may suppose that taking solutions of the form

$$
q=\hat{q}_{1} \delta(y-1)+\hat{q}_{2} \delta(y+1), \quad j=\hat{j}_{1} \delta(y-1)+\hat{j}_{2} \delta(y+1)
$$

has the potential to be a reasonable approximation to the full solution, at least away from marginality. Since this neglects the standing wave contribution that counteracts the two counter-propagating waves, we expect the resulting solutions have, compared to the solutions presented in figure 5 larger growth rates and a larger region of instability.

We start from the $(q, \eta)$ formulation with equation (2.6a) and (2.7). From the inversion relation (2.10), we have, in non-dimensional form,

$$
A_{1,2}=-\frac{1}{2 k}\left(\hat{j}_{1,2}+\hat{j}_{2,1} \mathrm{e}^{-2 k}\right), \quad \psi_{1,2}=-\frac{1}{2 k}\left(\hat{q}_{1,2}+\hat{q}_{2,1} \mathrm{e}^{-2 k}\right) .
$$

Our vorticity equation (2.6a) will have $\hat{j}$ involved so we also need expressions for $\hat{j}_{1,2}$. Using $A=\bar{B} \eta$ from (2.8), we obtain from equation (4.10)

$$
\hat{j}_{1,2}=-\frac{2 k}{1-\mathrm{e}^{-4 k}}\left(\eta_{1,2}-\eta_{2,1} \mathrm{e}^{-2 k}\right) .
$$

Substituting accordingly, the governing equations (2.6a) and (2.7) becomes

$$
\begin{aligned}
& \left(\frac{\partial}{\partial t} \pm \mathrm{i} k\right) \hat{q}_{1,2}= \pm \frac{\mathrm{i}}{2}\left(\hat{q}_{1,2}+\hat{q}_{2,1} \mathrm{e}^{-2 k}\right)-\frac{2 k M^{2}}{1-\mathrm{e}^{-4 k}}\left(\eta_{1,2}-\eta_{2,1} \mathrm{e}^{-2 k}\right), \\
& \left(\frac{\partial}{\partial t} \pm \mathrm{i} k\right) \eta_{1,2}=-\frac{\mathrm{i}}{2}\left(\hat{q}_{1,2}+\hat{q}_{2,1} \mathrm{e}^{-2 k}\right) .
\end{aligned}
$$

In writing equations (4.12), the fundamental assumption is that the other interface exists, which results in the appearance of exponential factors $\left(1-\mathrm{e}^{-4 k}\right)^{-1}$ multiplying some of the $\eta$ terms. This is unlike the stratified case considered in Rabinovich et al. (2011). Although the displacement is related to the perturbation magnetic potential and buoyancy for the respective cases, in the MHD case the current also appears in the vorticity equation, while there is no analogue of this in the stratified case. 
(a)

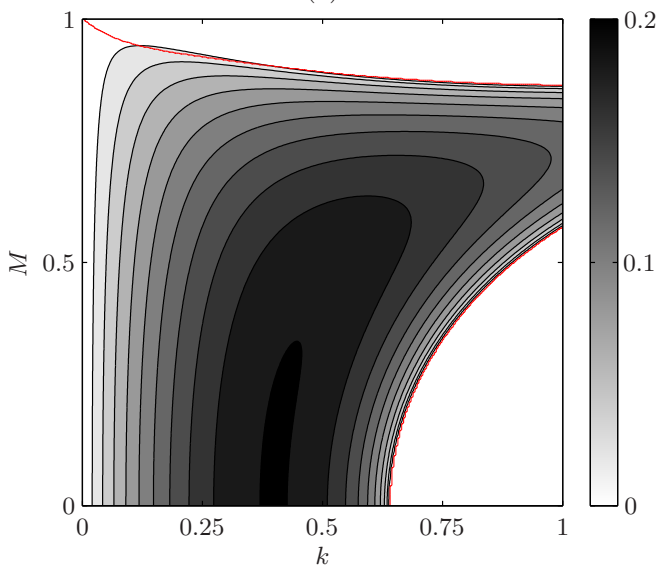

(b)

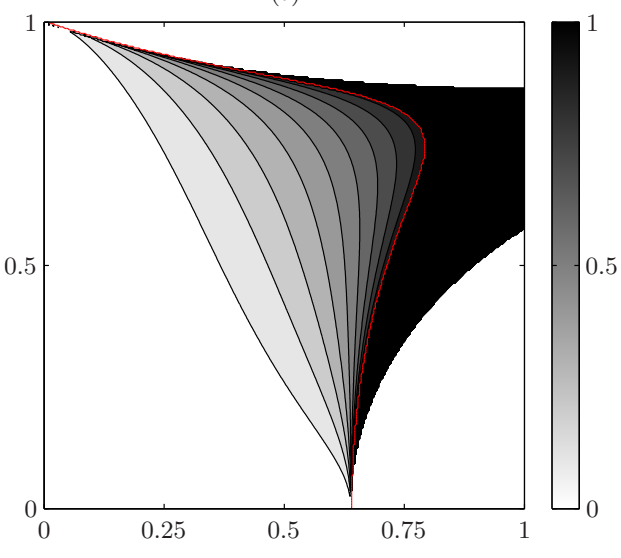

FIGURE 8. Results from taking only two interfaces, for the profile given in 4.5). (a) shows the growth rates $\left(k c_{i}\right)_{\text {int }}$ of the approximated solution from (4.13). (b) shows the weighted error $E=1-\left(k c_{i}\right)_{\text {full }} /\left(k c_{i}\right)_{\text {int }}$; the red line here is the stability boundary of the full solution in figure 5

Considering modal solutions, the system (4.12) has closed form solutions given by

$$
c= \pm \sqrt{1-\frac{1}{2 k}+\frac{1-\mathrm{e}^{-4 k}}{8 k^{2}}+M^{2} \pm \sqrt{\frac{1}{4 k^{2}}\left(1-\frac{1-\mathrm{e}^{-4 k}}{4 k}\right)^{2}+2 M^{2} \xi}},
$$

where

$$
\xi=1-\frac{1}{k}+\frac{1-\mathrm{e}^{-4 k}}{8 k^{2}}+\frac{1+\mathrm{e}^{-4 k}}{1-\mathrm{e}^{-4 k}} .
$$

When $M=0$, (4.13) reduces to the hydrodynamic solutions $c= \pm \sqrt{(1-2 k)^{2}-\mathrm{e}^{-4 k}} / 2 k$ (e.g., Drazin \& Reid 1981). For $k \ll 1$, a straight forward asymptotic analysis of (4.13) yields $c=\mathrm{i} \sqrt{1-M^{2}}$, which is the vortex sheet result in incompressible MHD (e.g., Chandrasekhar 1981).

Figure $8(a)$ shows the growth rates $\left(k c_{i}\right)_{\text {int }}$ of the unstable branch of (4.13). Like the full solution, the unstable modes here have $c_{r}=0$. In figure $8(b)$ we show contours of the weighted error $E=1-\left(k c_{i}\right)_{\text {full }} /\left(k c_{i}\right)_{\text {int }}$; when $E=0$, the approximated solution (4.13) completely agrees with the full solution, whilst $E=1$ shows the approximated solution predicts instability when it is otherwise absent in the full solution. In agreement with the hypotheses, we over-estimate growth rates as well as regions of instability, with the over-estimation being most significant in the short wave regime and near the stability boundary.

Previous authors analysing the stratified problem (Harnik et al. 2008; Rabinovich et al. 2011) have observed that, since there are two wave branches, the modal solutions will have a pro- and counter-propagating component. The existence of the two components are due to the fact that there is an intermediate step linking displacement with vorticity. In our schematic presented above in figure 7 , the instability results from the interaction of counter-propagating modes, so it is informative to investigate the role of the pro-propagating mode. A similar analysis to Rabinovich et al. (2011) may be carried out 
(a)

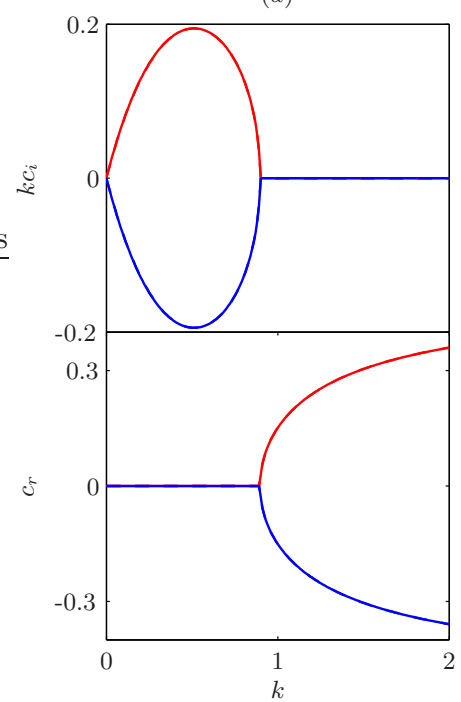

$(b)$

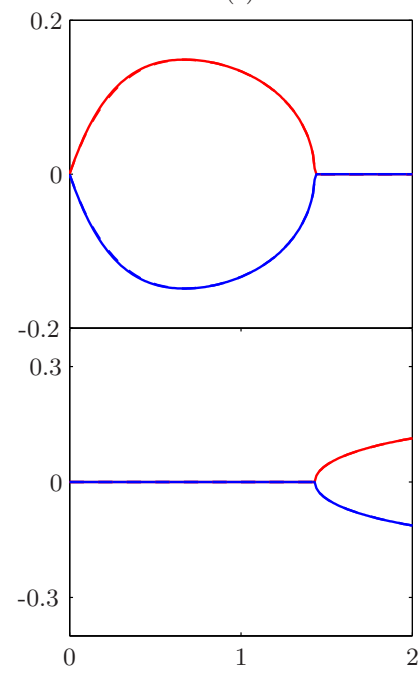

$(c)$

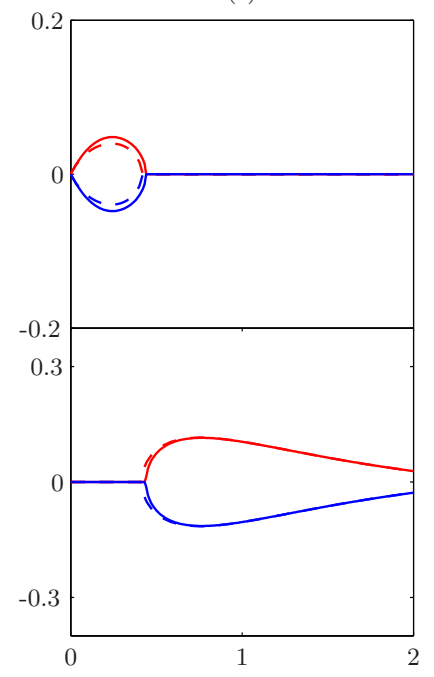

FiguRE 9. Line plots of the growth rate $k c_{i}$ (top row) and the phase speed $c_{r}$ (bottom row) associated with the growing and decaying solutions of the full solution 4.13), denoted by solid lines, and the $\chi=0$ solutions (4.16), denoted by dashed lines. These are evaluated at fixed $M$, with (a) $M=0.5,(b) M=0.75$, and (c) $M=0.9$. The $\chi=0$ solution is, for the most part, indistinguishable from the analytical solution.

here, by taking into account the asymmetric non-dimensional eigenstructure given by

$$
\hat{q}_{1}^{ \pm}=2 k c_{\mathrm{ra}}^{ \pm} \eta_{1}^{ \pm}, \quad \hat{q}_{2}^{ \pm}=-2 k c_{\mathrm{ra}}^{\mp} \eta_{2}^{ \pm}, \quad c_{\mathrm{ra}}^{ \pm}=-\frac{1}{4 k} \pm \sqrt{\left(\frac{1}{4 k}\right)^{2}+M^{2}} .
$$

We may transform the system of equations (4.12) into a system of equations in terms of $\left(\eta_{1}^{ \pm}, \eta_{2}^{ \pm}\right)$via a direct substitution, a self-similarity transform (e.g., Harnik et al. 2008), or otherwise. We come to essentially the same conclusions as Rabinovich et al. (2011), where, when there is an instability, the pro-propagating mode should satisfy the relation $\left(\eta_{1}^{+}, \eta_{2}^{-}\right)=-\chi\left(\eta_{2}^{+}, \eta_{1}^{-}\right)$, where $\chi \in[0,1]$, i.e., the pro-propagating mode on one flank is smaller by a factor of $\chi$ and in anti-phase with the counter-propagating mode on the other flank. The existence of the pro-propagating mode is to provide extra hindering to the counter-propagating modes, with its effect being most significant in the $k \ll 1$ regime. One could consider taking $\chi=0$ approximation that artificially removes the pro-propagating mode; the resulting analytical solution is

$$
c= \pm \sqrt{\left(1+c_{\mathrm{ra}}^{-}+\frac{M^{2} \mathrm{e}^{-4 k}}{\left(1-\mathrm{e}^{-4 k}\right)\left(c_{\mathrm{ra}}^{+}-c_{\mathrm{ra}}^{-}\right)}\right)^{2}-\left(c_{\mathrm{ra}}^{-}-\frac{2 k M^{2}}{1-\mathrm{e}^{-4 k}}\right)^{2}\left(\frac{\mathrm{e}^{-2 k}}{c_{\mathrm{ra}}^{+}-c_{\mathrm{ra}}^{-}}\right)^{2}} .
$$

We show in figure 9 several line plots of the analytical solution (4.13) and the $\chi=0$ solution (4.16). The differences between the solutions in this case are so slight that they are only distinguishable at high values of $M$. This points to the scenario that, although the pro-propagating mode must exist as part of the physics, its effect on the instability for this profile is almost negligible compared to the counter-propagating mode. One may obtain the numerical values of $\chi$ by computing the eigenfunctions (which are just complex numbers in this case), and taking $\chi=\left|\eta_{1}^{+}\right| /\left|\eta_{2}^{+}\right|=\left|\eta_{2}^{-}\right| /\left|\eta_{1}^{-}\right|$; although not shown here, we have $\chi \lesssim 0.1$ over most of the region where there is instability. 


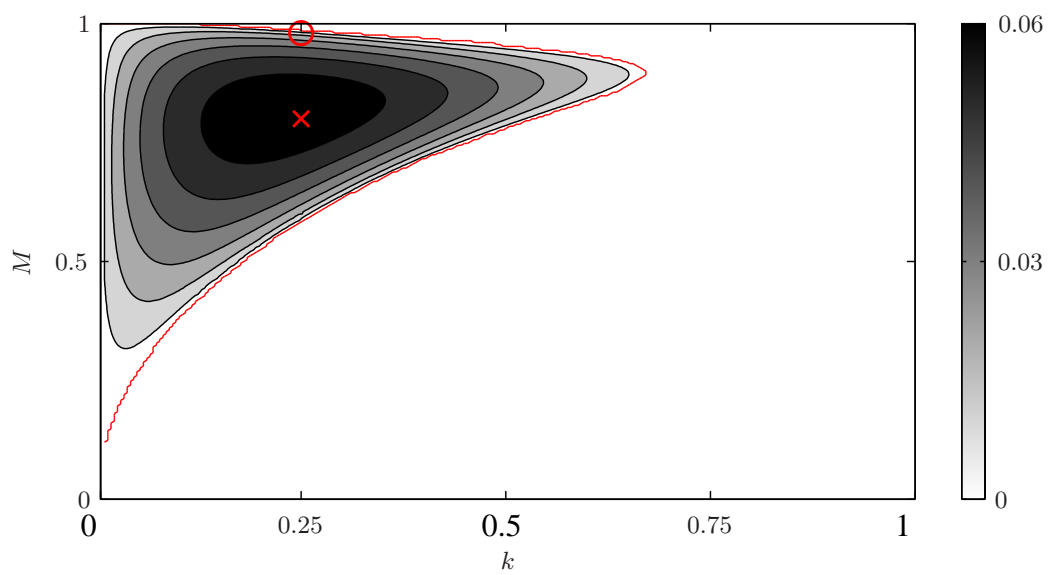

FIGURE 10. Growth rates $\left(k c_{i}\right)_{\text {full }}$ of the full numerical solution associated with the basic state (5.1). Here, $c_{r}=0$ for all unstable modes. The red cross and circle corresponds to the parameter location associated with the eigenfunction displayed in figure $11(a, b)$ respectively.

\section{Stable profile destabilised by a spatially varying magnetic field}

We now carry out a similar investigation for the case where a stable flow is destabilised by a spatially varying magnetic field. We consider the dimensional basic state

$$
\bar{U}(y)=\Lambda y, \quad \bar{B}(y)= \begin{cases}+\Gamma y, & y>L, \\ +\Gamma L, & |y|<L, \\ -\Gamma y, & y<-L,\end{cases}
$$

and $\partial \bar{Q} / \partial y=0$ here. Scaling by

$$
\tilde{B}=\Gamma L, \quad \tilde{T}=\frac{1}{\Lambda}, \quad \tilde{L}=L \quad \tilde{U}=\Lambda L,
$$

the non-dimensional parameter is again $M=\tilde{B} / \tilde{U}$, and $(\bar{B}, \bar{J}, j) \rightarrow M(\bar{B}, \bar{J}, j)$ after rescaling. This piecewise-linear magnetic field profile (resembling a wake) is chosen so that it satisfies $\bar{B} \Delta \bar{J}<0$. This profile choice is partly inspired by the parabolic magnetic field profiles $\bar{B} \sim y^{2}$ considered by Chen \& Morrison (1991) and Tatsuno \& Dorland (2006) and the single interface profile considered by Stern (1963). One notable difference however is that this profile has a well-defined region of maximum $|\partial \bar{J} / \partial y|$, whilst the profiles considered previously generally have $\partial \bar{J} / \partial y=$ const throughout the domain, so this profile is not intended to be directly comparable to those previous studies. This case also has some similarities to the problem where there is a linear shear with two density jumps (the Taylor-Caulfield type instability), considered previously by, for example, Rabinovich et al. (2011); we have here instead two jumps in the current profile. Again, $M=1$ is the cutoff for linear, normal mode instability.

Figure 10] shows contours of $\left(k c_{i}\right)_{\text {full }}$ for this instability problem, and, again, $c_{r}=0$ for the unstable modes. The shape of the instability region in parameter space may again be appropriately justified. At small $M$, waves are too slow to overcome the background advection except at $k \ll 1$. As $M$ increases, waves become sufficiently fast, overcome the background advection and achieve phase-locking. For large enough $M$, all waves are too fast to phase-lock.

In figure 11 we show two eigenfunctions, one that is representative of a growing mode 
(a)

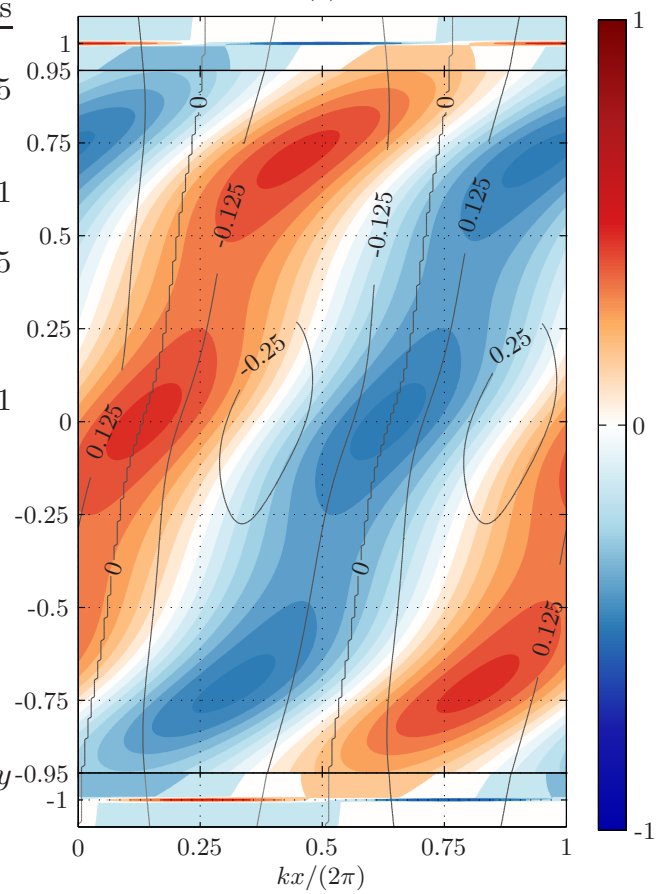

(b)

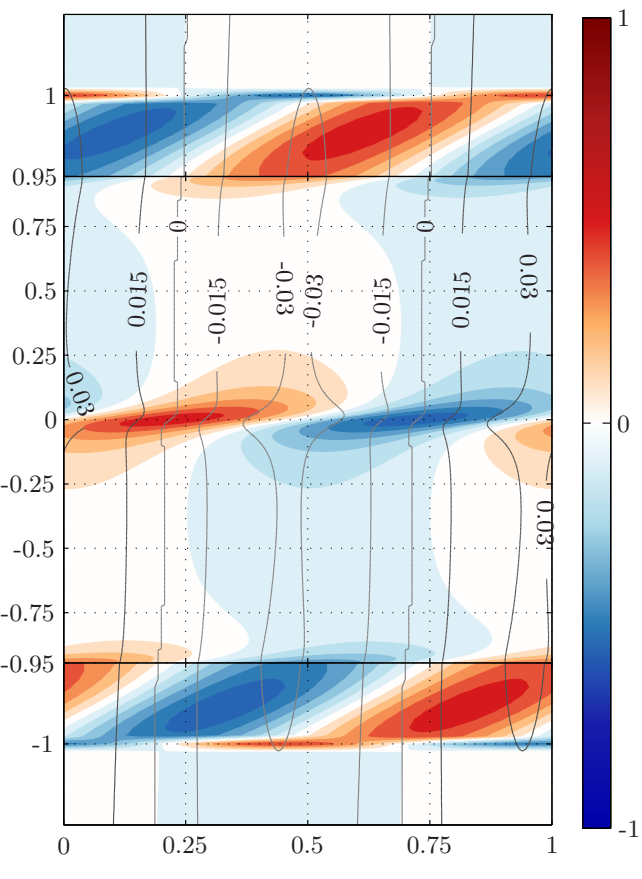

FiguRE 11. Representative eigenfunctions (normalised by the maximum absolute value of the vorticity) of the profile given in (5.1), both for $k=0.25$, but with (a) $M=0.8$, and (b) $M=0.98$. The red and blue shading are for positive and negative vorticity respectively, and the displacement is plotted as labelled contours. The $y$-scale is continuous between the panels (note the displacement contours are continuous in magnitude) but is not linear for display purposes.

away from the stability boundary, and one for a case close to marginality; again, the parameter locations are given respectively by the red cross and circle in figure 10 For both cases, we notice that the same contour levels are used for all three sections, unlike the previous instability problem displayed in figure 6. This immediately suggests that considering interfacial wave solutions only at $y= \pm 1$ as in (4.9) is going to be an overly drastic approximation, since we will be neglecting contributions that are comparable in size to those at the interface.

Like the previous case, we have significant structures appearing at $y= \pm M$, the locations where we have forced, stationary Alfvén waves. What is different in this case is that the schematic presented in figure 7 applies for the inner tilted structures, and so it is Alfvén waves at $y= \pm M$ rather than the waves supported by the interfaces that drive the instability. There are several extra interactions between the structures (affecting wave propagation and interaction) that lead to the overall instability eigenfunction in figure 11. Comparing between figure 11 $(a, b)$, we observe again that it is a mix of changes to the overall phase-shift between the structures that lead to the neutralisation of the instability. Notice also that, since we are approaching marginality by increasing $M$ in figure 11 (b), the displacement contours become increasingly in phase, as in the previous example in figure $6(b)$.

We considered decomposing the full vorticity eigenfunction into its constituents using (2.6a); in this case, it is only the $\bar{B}(\partial j / \partial x)$ and the $(\partial A / \partial x)(\partial \bar{J} / \partial y)$, divided by $\bar{U}-$ $c$, that contributes to the overall vorticity. Like the problem considered in $\S 4$, all the 
contributions away from $y= \pm 1$ come solely from the former term, whilst both contribute to the vorticity eigenfunction at the interfaces. It may be seen that while the latter term is associated with a particular contribution, the former term has associated a contribution that is in anti-phase and marginally larger than this, thus resulting in what is seen in figure 11] It is interesting in that although it looks like the contributions at $y= \pm 1$ plays a relatively minor role in the eigenfunction figure 11 as the cause for instability, it really must exist for the overall interaction resulting in the instability, via hindering/helping of the wave propagation over the domain.

We should stress here that the instability in figure 11 really does require the presence of two current jumps to operate, while the $\bar{B}(\partial j / \partial x)$ term acts to modify the resulting interaction. To support this claim, we have carried out calculations where we only have one jump. In this setting, there is no instability since the contributions on the other interface, its adjacent tilted structure and the standing wave in the centre are absent. The resulting vorticity eigenfunction has the interface and adjacent tilted structure in anti-phase, and there is no constructive interference there. Removing both the jumps (i.e., linear shear flow with a uniform background magnetic field) also does not result in instability. For the profile (5.1) we consider here, the strength of the current gradient and background magnetic field are simultaneously controlled by $M$. We considered a modified problem where the two parameters may be varied independently; we also arrive at similar conclusions to the one presented here.

We may take a similar approach to the work detailed in 4.3 , by neglecting contributions away from the interfaces, which results in analytical solutions that may be analysed accordingly. However, we do not expect this to provide an accurate approximation for this particular choice of basic state, since we are neglecting contributions that are of the same order of magnitude as the ones at the interface. A comparison of the full numerical solutions with the resulting analytical solution shows that the analytical solution grossly over-estimates growth rates and the region of instability. In light of the poor comparison of the analytical result with the correct full numerical solution, we omit here the results that are counterparts to those presented in $\$ 4.3$.

\section{Conclusion and discussion}

We have extended the interacting vorticity wave formalism to the MHD setting to provide a physical interpretation of the instability mechanism for MHD shear instabilities. In this framework, the existence of instability depends on whether the choice of basic state allows vorticity waves to resonate, and whether the field stabilises or destabilises is dependent on how the resulting configuration affects wave properties. We have demonstrated that vorticity generation occurs at the locations where the background magnetic field and the background shear are both non-zero so, unlike certain hydrodynamic cases, critical layers must play a role in the dynamics even for piecewise-linear basic states.

To demonstrate the modifications to the underlying instability mechanism by MHD effects, and to compare with previous results and to evaluate the limitations of the interfacial wave assumption, we considered the instability characteristics of two piecewiselinear basic states, one where the field stabilises the unstable flow, and the other where the field destabilises the stable flow. The first example considered is the Rayleigh profile. The growth rate contours of the full solution agrees with the previous results of Ray \& Ershkovich (1983); our contribution here is to rationalise the shape of the instability region via the properties of the phase-speeds of the wave propagation. Plots of the eigenfunctions shows that we effectively have a standing wave structure at $\bar{U}(y)-c=0$ in between two counter-propagating modes, and may be schematically represented by 
figure 7 Additionally, we are able to predict the phase relations of this standing wave contribution from the equations. As we approach marginality, one interesting feature is that we have additional structures appearing at the levels where $\bar{U}(y)-c= \pm M \bar{B}(y)$, and these are critical levels that correspond to locations where we have forced, stationary Alfvén waves. Changing the field strength affects both the strength of the critical layer and phase-shifts of the waves, and although there will be special cases where marginality is achieved when the critical layer contribution overwhelms the other contributions, generically speaking, it is a combination of the two effects that leads to neutralisation of the instability. In this example, we argued that the dynamics of two interfacial waves can serve as a reasonable approximation to the full problem. We predicted and found that such an approximation over-estimates the growth rate and the region of instability. Appropriate analyses in the manner of Rabinovich et al. (2011) were performed to explore the instability characteristics under the interfacial wave assumption.

The second example we considered is a linear shear flow destabilised by a spatially varying background magnetic field. The magnetic field profile was inspired by the parabolic profile $\bar{B}(y) \sim y^{2}$ considered by both Chen \& Morrison (1991) and Tatsuno \& Dorland (2006), although we stress that the results are not entirely comparable since $\partial \bar{J} / \partial y$ is constant throughout the domain for the parabolic profiles. With regards to the eigenfunction, a robust feature is that, like the previous example, tilted structures with significant contributions of vorticity exists at the critical levels. The schematic in figure 7 however applies instead to the forced, stationary Alfvén waves located at $\bar{U}(y)-c= \pm M \bar{B}(y)$ and is counteracted by the standing wave contribution associated with the critical level $\bar{U}(y)-c=0$. The principal interaction driving the instability are from these stationary Alfvén waves away from the interfaces rather than from interfacial waves.

Part of the reason for employing piecewise-linear profiles is to obtain a simplified problem for understanding the dynamics leading to instability, as well as for comparison with previous works on a similar topic. One important point we highlighted is that one needs to be careful when making the interfacial wave assumption, since vorticity generation is generally not localised. The non-local generation of vorticity occurs more generally when considering the instability problem for smooth basic states (arguably more realistic for modelling purposes), and the resulting eigenstructure generically has a spatial dependence on the cross-stream coordinate. Although waves are then not as welldefined, one may wonder whether the same mechanistic interpretation summarised by figure 7 here is schematically correct. One study that supports this was reported in one of the authors' $\mathrm{PhD}$ thesis $(\operatorname{Mak} 2013)$. For calculations of the profile $\bar{U}(y)=\tanh (y)$ and a uniform background magnetic field, plots of the eigenfunction showing structures similar to figure 6( $a$ ) here were found. We expect analogous structures to appear in eigenfunctions from calculations with other smooth basic states, demonstrating that shear instabilities may be interpreted as the mutual interaction of vorticity regions. One other possible scenario in smooth profiles is that the overall eigenfunction could look schematically like figure $4(b)$, occurring for example when the basic state gradients are weak/non-existent, although this has not been found for the examples considered here.

Although we have focussed on modal instabilities here, the formulation is kept in the $\partial \boldsymbol{\eta} / \partial t=\mathbf{A} \boldsymbol{\eta}$ form (where $\boldsymbol{\eta}$ denotes the state vector and $\mathbf{A}$ denotes the appropriate operator) so that it may also be used to investigate non-normal mode instabilities and transient growth (e.g., Constantinou \& Ioannou 2011; Guha \& Lawrence 2013).

Beyond incompressible MHD, this wave interaction framework, together with the previous works on for the stratified case (Harnik et al. 2008; Rabinovich et al. 2011; Carpenter et al. 2010, 2013; Guha \& Lawrence 2013) may perhaps explain the observations made in the previous work of Lecoanet et al. (2010), where they consider the shear 
instability problem in a stratified fluid, with a background magnetic field. The field in that case can stabilise or destabilise, and we suspect this is most likely due to whether the vorticity wave modes supported by the choice of the basic state can interact accordingly, leading to instability; we suspect this is why the Richardson number or Miles-Howard criterion (e.g., Miles 1961) is not necessarily applicable to stratified MHD shear flows.

Our profiles were chosen so that, when $M \geqslant 1,|B| \geqslant|U|$ pointwise everywhere, and a stability theorem forbids normal mode instabilities. Profiles violating this condition locally do suffer instabilities, and, in particular, have been found for studies in both twodimensional, spherical, incompressible and shallow water MHD (see the recent review by Gilman \& Callv 2007). Although this particular scenario is not one we have addressed here, a extension of our interpretation to spherical MHD appears possible (a hydrodynamic extension in spherical co-ordinates was given by Methven et al. 2005). These instabilities may be explained by energetic arguments, and an extension of our mechanistic interpretation will serve to complement the existing explanation. A formulation of the problem in spherical MHD allows for a more appropriate comparison to the existing results, and this is currently under investigation by the authors.

This work was initiated whilst JM and EH were visiting MISU, with EH supported by the Rossby visiting fellowship of the International Meteorological Institute of Sweden. EH is grateful to Michael Mond for fruitful discussion and to his teacher Alexander Ershkovich for his insight and wisdom. The order of authorship is alphabetical.

\section{Appendix A. Some formal analogies with the stratified problem}

Using (2.8) to rewrite (2.6a), the linearised vorticity equation in MHD is given by

$$
\left(\frac{\partial}{\partial t}+\bar{U} \frac{\partial}{\partial x}\right) q=-\frac{\partial \bar{Q}}{\partial y} \frac{\partial \psi}{\partial x}+\bar{B} \frac{\partial j}{\partial x}+\left(\bar{B} \frac{\partial \bar{J}}{\partial y}\right) \frac{\partial \eta}{\partial x} .
$$

For the analogous problem in the Bousinessq system, using the fact that $(\partial / \partial t+\bar{U} \partial / \partial x) b=$ $-w N^{2}(z)$ and $(\partial / \partial t+\bar{U} \partial / \partial x) \zeta=w$, integrating yields the relation that $b=-\eta N^{2}(z)$, where $N^{2}(z)$ is the background buoyancy frequency. The resulting vorticity equation reads

$$
\left(\frac{\partial}{\partial t}+\bar{U} \frac{\partial}{\partial x}\right) q=-\frac{\partial \bar{Q}}{\partial z} \frac{\partial \phi}{\partial x}-N^{2} \frac{\partial \zeta}{\partial x} .
$$

Then the formal similarity is that $-\bar{B}(\partial \bar{J} / \partial y) \leftrightarrow N^{2}$. The analogy is not entirely complete here because, as we have seen before, for $\bar{B}(\partial \bar{J} / \partial y)>0$, the destabilising effect of $\bar{B}(\partial \bar{J} / \partial y)$ is counteracted somewhat by the term $\bar{B}(\partial j / \partial x)$. In the case where we assume interfacial waves or plane waves, the analogy is augmented by relating $j$ to $A$ and then to $\eta$, which then gives $\bar{B}(\bar{B}-\Delta \bar{J} / 2 k) \leftrightarrow N^{2}$. However, as discussed in the text, isolated $\delta$-function solution neglects contributions away from the 'jump', and predicts instability when the global state (in the absence of a flow) should be stable (e.g., Lundquist 1951). We note, for completeness, gravity wave propagation and Rayleigh-Taylor instability has an analogous description in terms of waves and vorticity action, as in figure 4, by replacing current anomalies with buoyancy anomalies and considering the action of the resulting baroclinic torque (see also figure 2 and 3 of Harnik et al. 2008).

We note that, in the stratified problem, without assuming interfacial waves, the evolution equation for the perturbation energy is given by

$$
\frac{1}{2} \frac{\partial}{\partial t}\left\langle\left(u^{2}+w^{2}\right)+N^{2} \zeta^{2}\right\rangle=-\left\langle u w \frac{\partial \bar{U}}{\partial z}\right\rangle
$$


where the angle bracket denotes a domain integral. So if $N^{2}<0$, it is still possible for the perturbation kinetic energy to grow even in the absence of a background flow. In the MHD case, a similar manipulation leads first to

$$
\frac{\partial u}{\partial t}+\bar{U} \frac{\partial u}{\partial x}=-v \frac{\partial \bar{U}}{\partial y}-\frac{\partial}{\partial x}(\cdots), \quad \frac{\partial v}{\partial t}+\bar{U} \frac{\partial v}{\partial x}=\bar{B}\left(\frac{\partial \bar{J}}{\partial y} \eta+j\right)-\frac{\partial}{\partial y}(\cdots),
$$

where the terms in $(\cdots)$ between the two equations are the same. Again, it is the appearance of the perturbation current that makes the analogy incomplete. If we however assume that $\partial \bar{J} / \partial y=\Delta \bar{J} \delta(y-L)$ and $j=\hat{j} \mathrm{e}^{\mathrm{i} k x} \delta(y-L)$, then it may be shown that, at $y=L$,

$$
\frac{1}{2} \frac{\partial}{\partial t}\left\langle\left(u^{2}+v^{2}\right)+\bar{B}(2 k \bar{B}-\Delta \bar{J}) \eta^{2}\right\rangle=-\left\langle u v \frac{\partial \bar{U}}{\partial y}\right\rangle
$$

and, in the absence of a background flow, if $\bar{B} \Delta \bar{J}>0$, it is still possible for the perturbation kinetic energy to grow at the expense of magnetic energy, resulting in instability.

With regards to the non-dimensional units, we recall that, in the stratified case, the governing non-dimensional parameter is $R i=N^{2} /(\partial \bar{U} / \partial z)^{2}$. In MHD, the governing non-dimensional number is

$$
M^{2}=\frac{B_{0}^{2}}{U_{0}^{2}} \sim \frac{\bar{B}\left(L^{2} \bar{B}\right)}{L^{2} \bar{U}^{2}} \sim \frac{-\bar{B}(\partial \bar{J} / \partial y)}{(\partial \bar{U} / \partial y)^{2}},
$$

and so $M^{2}$ has a formal analogy with $R i$. We should stress that this is only a formal analogy, since it does not take into account of the $\bar{B}(\partial j / \partial x)$ term, which results in wave modes that are fundamentally different from the gravity wave modes. We note that, in a similar vein, Stern (1963) defines what he calls the magnetic Richardson number as (translated into our notation) $R i_{m}=(\partial \bar{B} / \partial y)^{2} /(\partial \bar{U} / \partial y)^{2}$.

\section{Appendix B. Formulation in terms of $q$ and $j$ variables}

One alternative approach is to use $q$ and $j$ as the fundamental variable over $q$ and $\eta$ when considering interface solutions. One immediate issue is that, from the current perturbation equation (2.11), $\bar{Q}$ and $\bar{J}$ are not-well defined at the interface locations. A further approximation that one might make is that the terms with the coefficient $\bar{Q}$ and $\bar{J}$ are small compared to $\partial \bar{Q} / \partial y$ and $\partial \bar{J} / \partial y$; this may be appropriate if we have reason to believe that interfacial waves are the most important aspect to the dynamics, perhaps when the basic state gradients are strong. With this assumption, we obtain the system of equations

$$
\begin{aligned}
& \left(\frac{\partial}{\partial t}+\bar{U} \frac{\partial}{\partial x}\right) q=-\frac{\partial \bar{Q}}{\partial y} \frac{\partial \psi}{\partial x}+\bar{B} \frac{\partial j}{\partial x}+\frac{\partial \bar{J}}{\partial y} \frac{\partial A}{\partial x} \\
& \left(\frac{\partial}{\partial t}+\bar{U} \frac{\partial}{\partial x}\right) j=+\frac{\partial \bar{Q}}{\partial y} \frac{\partial A}{\partial x}+\bar{B} \frac{\partial q}{\partial x}-\frac{\partial \bar{J}}{\partial y} \frac{\partial \psi}{\partial x}
\end{aligned}
$$

With this, we notice that, if we define a generalised streamfunction and vorticity as $\phi^{ \pm}=(\psi \pm A) / 2$ and $\mathcal{Q}^{ \pm}=(q \pm j) / 2$, then we have $\nabla^{2} \phi^{ \pm}=Q^{ \pm}$and that

$$
\left[\frac{\partial}{\partial t}+(\bar{U} \mp \bar{B}) \frac{\partial}{\partial x}\right] \mathcal{Q}^{ \pm}=-\left(\frac{\partial \bar{Q}}{\partial y} \pm \frac{\partial \bar{J}}{\partial y}\right) \frac{\partial \phi^{\mp}}{\partial x} .
$$

This has some formal similarities to employing Elsasser variables (e.g., Biskamp 2003) in writing the MHD equations, although this is of course different since we have made an approximation by dropping certain terms in the $j$ equation. 


\section{REFERENCES}

Baines, P. G. \& Mitsudera, H. 1994 On the mechanism of shear instabilities. J. Fluid Mech. 276, 327-342.

Balmforth, N. J., Roy, A. \& Caulfield, C. P. 2012 Dynamics of vorticity defects in stratified shear flow. J. Fluid Mech. 694, 292-331.

Biskamp, D. 2003 Magnetohydrodynamic turbulence. Cambridge University Press.

Bretherton, F. P. 1966 Baroclinic instability and the short wavelength cut-off in terms of potential vorticity. Q. J. Roy. Met. Soc. 92, 335-345.

CAIRns, R. A. 1979 The role of negative energy waves in some instabilities of parallel flows. $J$. Fluid Mech. 92, 1-14.

Carpenter, J. R., Balmforth, N. J. \& Lawrence, G. A. 2010 Identifying unstable modes in stratified shear layers. Phys. Fluids 22, 054104.

Carpenter, J. R., Tedford, E. W., Heifetz, E. \& Lawrence, G. A. 2013 Instability in stratified shear flow: Review of a physical interpretation based on interacting waves. Appl. Mech. Rev. 64, 061001.

Caulfield, C. P. 1994 Multiple linear instability of layered stratified shear flow. J. Fluid Mech 258, 255285.

Chandrasekhar, S. 1981 Hydrodynamic and hydromagnetic stability, dover edn. Dover Publications Inc.

Chen, X. L. \& Morrison, P. J. 1991 A sufficient condition for the ideal instability of shear flow with parallel magnetic field. Phys. Fluids B 3, 863865.

Constantinou, N. C. \& Ionnnou, P. J. 2011 Optimal excitation of two-dimensional Holmboe instabilities. Phys. Fluids 23, 074102.

Drazin, P. G. \& ReID, W. H. 1981 Hydrodynamic stability, 2nd edn. Cambridge University Press.

Gilman, P. A. \& Cally, P. S. 2007 Global MHD instabilities of the tachocline. In The Solar Tachocline (ed. D. W. Hughes, R. Rosner \& N. O. Weiss). Cambridge University Press.

Gilman, P. A. \& Fox, P. A. 1997 Joint instability of latitudinal differential rotation and toroidal magnetic fields below the solar convection zone. Astrophys. J. 484, 439-454.

Goldston, R. J. \& Rutherford, P. H. 1995 Introduction to plasma physics, pap/dskt edn. Taylor \& Francis.

Guha, A. \& Lawrence, G. A. 2013 A wave interaction approach to studying non-modal homogeneous and stratified shear instabilities. J. Fluid Mech. 755, 336-364.

Harnik, N., Heifetz, E., Umurhan, O. M. \& Lott, F. 2008 A buoyancy-vorticity wave interaction approach to stratified shear flow. J. Atmos. Sci. 65, 2615-2630.

Heifetz, E., Bishop, C. H., Hoskins, B. J. \& Methven, J. $2004 a$ The counter-propagating Rossby-wave perspective on baroclinic instability. I: Mathematical basis. Q. J. Roy. Met. Soc. 130, 211-231.

Heifetz, E., Methven, J., Hoskins, B. J. \& Bishop, C. H. $2004 b$ The counter-propagating Rossby-wave perspective on baroclinic instability. II: Application to the Charney model. Q. J. Roy. Met. Soc. 130, 233-258.

Holmboe, J. 1962 On the behaviour of symmetric waves in stratified shear layers. Geophys. Publ. 24, 67-113.

Hoskins, B. J., McIntyre, M. E. \& Robertson, A. W. 1985 On the use and significance of isentropic potential vorticity maps. Q. J. Roy. Met. Soc. 111, 877-946.

Hughes, D. W. \& Tobias, S. M. 2001 On the instability of magnetohydrodynamic shear flows. Proc. R. Soc. Lond. A 457, 1365-1384.

Kent, A. 1966 Instability of laminar flow of a perfect magnetofluid. Phys. Fluids 9, 1286.

KEnt, A. 1968 Stability of laminar magnetofluid flow along a parallel magnetic field. J. Plasma Phys. 2, 543-556.

Leconnet, D., Zweibel, E. G., Townsend, R. H. D. \& Huang, Y.-M. 2010 Violation of Richardson's criterion via introduction of a magnetic field. Astrophys. J. 712, 1116-1128.

Lотт, F. 2003 Large-scale flow response to short gravity waves breaking in a rotating shear flow. J. Atmos. Sci. 1704, 1691-4862.

Lundquist, S. 1951 On the stability of magneto-hydrostatic fields. Phys. Rev. 83, 307-311. 
MAK, J. 2013 Shear instabilities in shallow-water magnetohydrodynamics. PhD thesis, University of Leeds.

Methven, J., Heifetz, E., Hoskins, B. J. \& Bishop, C. H. 2005 The counter-propagating Rossby-wave perspective on baroclinic instability. III: Primitive-equation disturbances on the sphere. Q. J. Roy. Met. Soc. 131, 1393-1424.

Miles, J. W. 1961 On the stability of heterogeneous shear flows. J. Fluid Mech. 10, 496-508.

Rabinovich, A., Umurhan, O. M., Harnik, N., Lott, F. \& Heifetz, E. 2011 Vorticity inversion and action-at-a-distance instability in stably stratified shear flow. J. Fluid Mech. 670, 301-325.

Ray, T. P. \& Ershkovich, A. I. 1983 Kelvin-Helmholtz instabilities in a sheared compressible plasma. Mon. Not. R. Astron. Soc. 204, 821-831.

Ruderman, M. S \& Belov, N. A 2010 Stability of MHD shear flows: Application to space physics. J. Phys.: Conf. Ser. 216, 012016.

Stern, M. E. 1963 Joint instability of hydromagnetic fields which are separately stable. Phys. Fluids 6, 636-642.

Tatsuno, T. \& Dorland, W. 2006 Magneto-flow instability in symmetric field profiles. Phys. Plasmas 13, 092107.

TAYLOR, G. I. 1931 Effect of variation in density on the stability of superposed streams of fluid. Proc. R. Soc. Lond. A 132, 499-523. 